\title{
Scalable One-Pot - Liquid-Phase Oligonucleotide Synthesis for Model Network Hydrogels
}

\section{Supporting Information}

Guido Creusen,a, a,b Cecilia Oluwadunsin Akintayo, ${ }^{\mathrm{a}, \mathrm{b}, \mathrm{c}, \mathrm{d}}$ Katja Schumann, ${ }^{\mathrm{a}}$ Andreas Walthera,b,c,d*

${ }^{a} A^{3}$ BMS Lab, Institute for Macromolecular Chemistry, University of Freiburg, Stefan-Meier-Straße 31, 79104 Freiburg, Germany

${ }^{\mathrm{b}}$ Freiburg Materials Research Center, University of Freiburg, Stefan-Meier-Straße 21, 79104 Freiburg, Germany

${ }^{\mathrm{C}}$ Freiburg Center for Interactive Materials and Bioinspired Technologies, University of Freiburg, GeorgesKöhler-Allee 105, 79110 Freiburg, Germany

'DFG Cluster of Excellence "Living, Adaptive and Energy-Autonomous Materials Systems" (livMatS), 79110 Freiburg, Germany.

*andreas.walther@makro.uni-freiburg.de

\section{Table of contents}

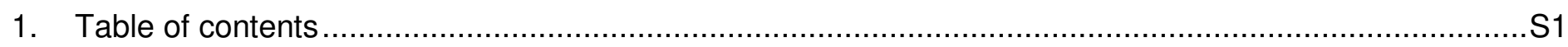

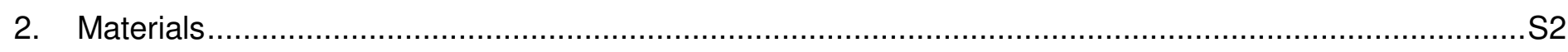

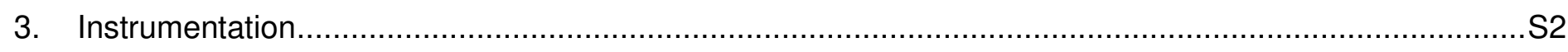

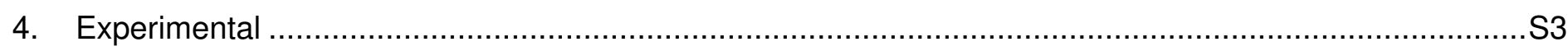

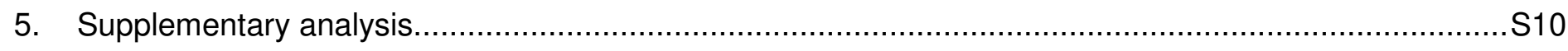




\section{Materials}

DMT-dT phosphoramidite, DMT-dA(Bz) phosphoramidite, 5-(Ethylthio)-1H-tetrazole (ETT activator, $250 \mathrm{mM}$ in anhydrous acetonitrile), meta-chloroperbenzoic acid (mCPBA) $\leq 77 \%$, tert-butyl hydroperoxide (TBHP) solution 5.0-6.0 M in decane, TBHP solution $70 \%$ in $\mathrm{H}_{2} \mathrm{O}$, dichloroacetic acid (DCA) $\geq 99 \%$, triethyl silane (TES) $99 \%$, sodium phosphate dibasic dehydrate $\geq 99 \%$, ethylenediaminetetraacetic acid (EDTA) $\geq 99 \%$ were purchased from Sigma-Aldrich. Amonnia 32\% in water, methylamine $40 \%$ in water, acetonitrile (HPLC gradient grade), 2propanol (analytical grade), acetic acid 100\%, dichloromethane (DCM) $\geq 99 \%$ were purchased from Merck Millipore. Acetonitrile anhydrous, trichloroacetic acid (TCA) 99\% were purchased from ABCR GmbH. Triethylamine 99\%, diethyl ether (analytical grade) were purchased from Carl Roth. Regenerated Cellulose Dialysis membrane SpectraPor-1 6-8 kDa MWCO was purchased from Spectrum Chemical. Deuterated solvents for NMR analysis were purchased from Deutero $\mathrm{GmbH}$. Filter paper $1670150 \mathrm{~mm}$ was purchased from Macherey-Nagel. 4-arm PEG-OH $\left(M_{\mathrm{n}, \mathrm{MALDI}}=41,170 \mathrm{~g} / \mathrm{mol}\right)$ was purchased from JenKem Technology USA.

\section{Instrumentation}

HPLC (analytical) was measured using a Dionex UltiMate 3000 system with a VWD-3400 RS Variable Wavelength Detector (set to $260 \mathrm{~nm}$ ) with a $10 \mathrm{~mm}$ flow cell. Chromatography was performed using a ThermoFischer DNAPac RP column $\left(100 \times 3.0 \mathrm{~mm}, 4 \mu \mathrm{m}\right.$ particle size) at $40{ }^{\circ} \mathrm{C}$, running a gradient with a flow rate of $0.4 \mathrm{~mL} / \mathrm{min}$ from $100 \% A$ and $0 \% B$ to $70 \% A$ and $30 \% B$ in 20 min followed by $10 \% A$ and $90 \%$ in 5 min, where it stays 5 more minutes. Solvent $A: 5 \%$ acetonitrile, $95 \% 100 \mathrm{mM}$ triethylamine/acetic acid pH 7.8 in water. Solvent B: acetonitrile. All samples were injected with $100 \mu \mathrm{L}$. The UV traces at $260 \mathrm{~nm}$ were subtracted by the UV trace of an injection of $100 \mu \mathrm{L}$ milliQ water, and then normalized. Samples were prepared by deprotecting at $20 \mathrm{mg} / \mathrm{mL}$ with concentrated ammonia/methylamine (AMA) solution (30 min for 4-arm PEG-T, 120 min for 4arm PEG- $A_{n}$ ), then diluted 200-fold with phosphate buffer (pH 8.0, 75 mM phosphate, 1 mM EDTA, 150 mM Na+).

HPLC (preparative) was performed using a Dionex UltiMate 3000 system with a VWD-3400 Variable Wavelength Detector (set to $260 \mathrm{~nm}$ ) with a $0.4 \mathrm{~mm}$ flow cell. Chromatography was performed using a Hamilton PRP-3 column ( $250 \times 21.2 \mathrm{~mm}, 12-20 \mu \mathrm{m}$ particle size), running a gradient with a flow rate of $20 \mathrm{~mL} / \mathrm{min}$ from $100 \% A$ and $0 \% B$ to $70 \% A$ and $30 \% B$ in $10 \mathrm{~min}$, followed by $20 \% A$ and $80 \% B$ in 5 min, where it stays for 3 min. Solvent A: 5\% acetonitrile, 95\% $100 \mathrm{mM}$ triethylamine/acetic acid pH 7.8 in water. Solvent B: acetonitrile. Samples were prepared by deprotecting at $100 \mathrm{mg} / \mathrm{mL}$ in AMA solution (30 min for 4-arm PEG-T, $120 \mathrm{~min}$ for 4-arm PEG-An), followed by diluting 1-fold with milliQ water. After purification, the collected fraction was dialyzed (6-8 kDa MWCO RC membrane) against phosphate buffer (pH 8.0, $75 \mathrm{mM}$ phosphate, $150 \mathrm{mM} \mathrm{Na}$ ), dialyzed against milliQ water, and isolated by lyophilization. 
Size exclusion chromatography (SEC) was performed using an Agilent 1200 system equipped with a 1260 Infinity pump and a refractive index detector. One pre-column and three GRAM gel columns $(8 \times 300 \mathrm{~mm}$, Polymer Standards Service) with pore sizes $30,1000,1000 \AA$ were used at $50{ }^{\circ} \mathrm{C}$ and a flow rate of $1 \mathrm{~mL} / \mathrm{min}$ DMAc $+0.5 \% \mathrm{LiBr}$. Narrowly distributed poly(methyl methacrylate) standards were used for calibration (Polymer Standards Service).

Nuclear magnetic resonance (NMR) spectroscopy was measured at $300 \mathrm{MHz}$ on a Bruker ARX 300 spectrometer. Chemical shifts are shown relative to the residual solvent peaks $\left({ }^{1} \mathrm{H}\right)$ and $\mathrm{H}_{3} \mathrm{PO}_{4}\left({ }^{31} \mathrm{P}\right)$.

UV-Vis measurements for DNA concentration determination were done using a ScanDrop spectrometer and a Hellma TrayCell with a path length of $1.0 \mathrm{~mm}$, using the absorption at $260 \mathrm{~nm}$ and the averaged extinction coefficients.

Agarose gel electrophoresis (AGE) was conducted using a 20-sample gel chamber and a constant voltage of $80 \mathrm{~V}$ for $60 \mathrm{~min}$. Agarose gel was prepared $15 \mathrm{~g} / \mathrm{L}$ in tris-acetate EDTA buffer, and Roti-GelStain was added right before casting the gel with a mixing ratio of $20 \mu \mathrm{L}$ per $100 \mathrm{~mL}$ agarose solution. Samples were prepared by deprotecting at $20 \mathrm{mg} / \mathrm{mL}$ with concentrated ammonia/methylamine (AMA) solution (30 min for 4-arm PEG-Th, 120 min for 4-arm PEG- $A_{n}$ ), diluting 10-fold with milliQ water and freeze drying, then dissolving $2 \mathrm{mg} / \mathrm{mL}$ in trisacetate buffer with $20 \%$ glycerol. Images were captured by irradiating with UV light and using a blue band-pass filter.

Rheology was measured using an Anton-Paar MCR-301 rheometer with a $25 \mathrm{~mm}$ plate-plate geometry.

\section{Experimental}

\section{Literature procedure - coupling step (4-arm PEG ${ }_{41 \mathrm{k}}$-dT-DMT (P'II))}

To vacuum-dried 4-arm PEG-OH (2.010 g, $48.8 \mu \mathrm{mol}$ polymer, $195 \mu \mathrm{mol}$ OH-groups), solutions of $250 \mathrm{mM}$ ETT activator (12 eq. to OH-groups, $2.343 \mathrm{mmol}, 9.37 \mathrm{~mL}$ ) and $100 \mathrm{mM} \mathrm{DMT-dT}$ phosphoramidite (3 eq. to OHgroups, $586 \mu \mathrm{mol}, 5.86 \mathrm{~mL}$ ) in dry acetonitrile were added. The solution was stirred for 60 minutes, and then precipitated into diethyl ether. The product was filtered and washed with ether, and then re-precipitated 3 times from a $10 \%$ solution in DCM into diethyl ether. The product was dried under high vacuum overnight. Samples were collected after each precipitation/washing step. ${ }^{1} \mathrm{H}-\mathrm{NMR}$ and ${ }^{31} \mathrm{P}-\mathrm{NMR}$ in Figure S1 and Figure S11.

\section{Literature procedure - oxidation step anhydrous TBHP (4-arm PEG $_{41 k}$-dT-DMT $\left(P^{V}\right)$ )}

4-arm PEG-dT-DMT (PIII) isolated after the coupling step was dissolved in acetonitrile at a concentration of 150 $\mathrm{mg} / \mathrm{mL}$ (535 mg, $12.2 \mu \mathrm{mol}$ polymer, $48.9 \mu \mathrm{mol}$ phosphite groups). The reaction mixture was stirred in an ice 
bath, and tert-butyl hydroperoxide (50 eq. to phosphite groups, $2.45 \mathrm{mmol}, 0.445 \mathrm{~mL}$ ) was added as a solution in decane. Samples were collected in time intervals by precipitating into cold diethyl ether followed by washing with diethyl ether and drying under vacuum. ${ }^{1} \mathrm{H}-\mathrm{NMR}$ and ${ }^{31} \mathrm{P}-\mathrm{NMR}$ in Figure S2, oxidation kinetics in Figure S4. Note that the anhydrous TBHP was commercially available as a 5.0-6.0 M solution in decane, so we added the solution assuming a concentration of $5.5 \mathrm{M}$.

\section{Literature procedure - oxidation step aqueous TBHP (4-arm PEG $_{41 k}-d T-D M T\left(P^{V}\right)$ )}

4-arm PEG-dT-DMT ( $\mathrm{P}^{\mathrm{III}}$ ) isolated after the coupling step (487 mg, $11.1 \mu \mathrm{mol}$ polymer, $44.5 \mu \mathrm{mol}$ phosphite groups) was dissolved in acetonitrile at a concentration of $150 \mathrm{mg} / \mathrm{mL}$. The reaction mixture was stirred at room temperature, and cold tert-butyl hydroperoxide (10 eq. to phosphite groups, $445 \mu \mathrm{mol}, 57 \mu \mathrm{L}$ ) was added as a $70 \%$ solution in water. Samples were collected in time intervals by precipitating into cold diethyl ether followed by washing with diethyl ether and drying under vacuum. ${ }^{31} \mathrm{P}-\mathrm{NMR}$ and oxidation kinetics in Figure S4.

\section{Literature procedure - detritylation step (4-arm PEG $_{41 \mathrm{k}}-\mathrm{dT}-\mathrm{OH}$ )}

4-arm PEG-dT-DMT ( $\mathrm{P}^{\vee}$ ) (520 mg, $11.9 \mu \mathrm{mol}$ polymer, $47.5 \mu \mathrm{mol}$ phosphite groups) isolated after the oxidation step with TBHP was dissolved $50 \mathrm{mg} / \mathrm{mL}$ polymer in $30 \mathrm{mg} / \mathrm{mL}$ TCA solution in DCM. The mixture turned to an intense orange color immediately, and the color remained unchanged throughout the detritylation. Samples were collected in time intervals by precipitating into cold diethyl ether followed by washing with diethyl ether, then reprecipitating from DCM into diethyl ether and drying under vacuum. After 60 minutes the entire batch was precipitated into cold diethyl ether, and re-precipitated from DCM into diethyl ether. The product was dried under vacuum. ${ }^{1} \mathrm{H}-\mathrm{NMR}$ in Figure $\mathbf{1 h}$, kinetics in Figure $\mathbf{1 i}$.

As this product still had ca. 4\% DMT-groups left, the product was dissolved at $50 \mathrm{mg} / \mathrm{mL}$ again in $30 \mathrm{mg} / \mathrm{mL}$ TCA solution in DCM, and after 15 minutes precipitated into cold diethyl ether, washed with diethyl ether, reprecipitated from DCM into diethyl ether, and dried under vacuum. ${ }^{1} \mathrm{H}$-NMR and ${ }^{31} \mathrm{P}-\mathrm{NMR}$ of the DMT-off product in Figure S3.

\section{One-pot coupling-oxidation experiments (dT)}

As an example run (described here for aqueous TBHP), to vacuum-dried 4-arm PEG-OH (1.502 g, $36.5 \mu \mathrm{mol}$ polymer, $146 \mu \mathrm{mol} O H$-groups) was added $250 \mathrm{mM}$ ETT activator (12 eq. to OH-groups, $1.75 \mathrm{mmol}, 7.00 \mathrm{~mL}$ ) and $100 \mathrm{mM}$ DMT-dT phosphoramidite (3 eq. to OH-groups, $438 \mu \mathrm{mol}, 4.38 \mathrm{~mL}$ ) in dry acetonitrile. After 60 minutes, $1.0 \mathrm{~mL}$ of sample were collected by precipitating twice into cold 2-propanol and washing with diethyl ether. The reaction mixture contained $33.6 \mu \mathrm{mol}$ polymer and $403 \mu \mathrm{mol} \mathrm{P}^{\mathrm{III}}$ after collecting the sample. TBHP 
was then added as a $70 \%$ solution in water (10 eq. to PIII $4.04 \mathrm{mmol}, 0.52 \mathrm{~mL}$ ). Throughout the oxidation, a total of $6.0 \mathrm{~mL}$ of samples were collected in time intervals by precipitating twice into cold 2-propanol and washing with diethyl ether. ${ }^{1} \mathrm{H}-\mathrm{NMR},{ }^{31} \mathrm{P}-\mathrm{NMR}$ and oxidation kinetics in Figure S5 and Figure S6.

Note that samples need to be precipitated twice when the DMT-groups are still attached. The experiment was conducted in a similar fashion with anhydrous TBHP (50 eq. to PIII, in ice bath) (Figure 1e-f).

\section{$t$-BuOH side reaction experiment}

First, 4-arm PEG-OH was coupled with 3 eq. of DMT-dT phosphoramidite (as a $100 \mathrm{mM}$ solution in dry acetonitrile) and 12 eq. of ETT (as a $250 \mathrm{mM}$ solution in dry acetonitrile) for 60 minutes (34.5 $\mu \mathrm{mol}$ OH-groups, $103.4 \mu \mathrm{mol}$ phosphoramidite, $413.6 \mu \mathrm{mol}$ ETT). After the coupling step, tert-butanol was added without prior purification (50 eq. to PIII, $5.17 \mathrm{mmol}, 0.494 \mathrm{~mL}$ ). After 60 minutes the reaction mixture was precipitated precipitating twice into cold 2-propanol, washed with 2-propanol, and washed with diethyl ether. ${ }^{1} \mathrm{H}-\mathrm{NMR}$ and ${ }^{31} \mathrm{P}-\mathrm{NMR}$ in Figure S7.

\section{One-pot coupling-oxidation-detritylation (dT)}

To vacuum-dried 4-arm PEG-OH (1.500 g, $36.4 \mu \mathrm{mol}$ polymer, $146 \mu \mathrm{mol}$ OH-groups), ETT in (250 mM in dry acetonitrile 12 eq. to $\mathrm{OH}$-groups, $1.75 \mathrm{mmol}, 7.00 \mathrm{~mL}$ ) and DMT-dT phosphoramidite (100 mM in dry acetonitrile, 3 eq. to $\mathrm{OH}$-groups, $437 \mu \mathrm{mol}, 4.37 \mathrm{~mL}$ ) were added. After 60 minutes, $1.0 \mathrm{~mL}$ of sample were collected by precipitating twice into cold 2-propanol and washing with diethyl ether. After collecting the sample, the reaction

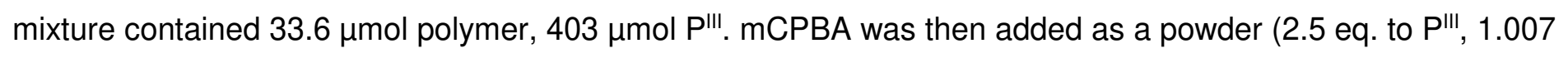
$\mathrm{mmol}, 0.226 \mathrm{~g}$ ). Throughout the oxidation, a total of $3.0 \mathrm{~mL}$ of samples were collected in time intervals by precipitating twice into cold 2-propanol and washing with diethyl ether. To the remaining reaction mixture (now $25.0 \mu \mathrm{mol}$ polymer, $300 \mu \mathrm{mol}$ DMT-groups) was added TCA as a $600 \mathrm{mg} / \mathrm{mL}$ solution in DCM (25 eq. to DMTgroups, $7.50 \mathrm{mmol}, 2.04 \mathrm{~mL}$ ) and TES (15 eq. to DMT-groups, $4.50 \mathrm{mmol}, 0.72 \mathrm{~mL}$ ). Samples were collected in time intervals by precipitating twice into cold 2-propanol and washing with diethyl ether. After the mixture turned colorless (9 $\mathrm{min}$ ), the sample was precipitated once into cold 2-propanol, washed with 2-propanol and then with diethyl ether. ${ }^{1} \mathrm{H}-\mathrm{NMR}$ and ${ }^{31} \mathrm{P}-\mathrm{NMR}$ in Figure $\mathbf{5 9 .}$

Note that mCPBA was commercially available as a solid of $\leq 77 \%$, so we added the amount assuming a mCPBAcontent in the powder of $77 \%$. Samples were precipitated twice when the DMT-groups were still (partially) on. The final DMT-off product was only precipitated and washed once. 


\section{One-pot coupling-oxidation-detritylation (dA) and depurination experiments}

An example run for 60 eq. TCA / 25 eq. TES at $0{ }^{\circ} \mathrm{C}$ is described here. Vacuum-dried 4-arm PEG-OH $(2.000 \mathrm{~g}$, $48.8 \mu \mathrm{mol}$ polymer, $195.1 \mu \mathrm{mol} \mathrm{OH}$-groups) was dissolved by adding ETT activator (250 mM in dry acetonitrile, 12 eq. to $\mathrm{OH}$-groups, $2.34 \mathrm{mmol}, 9.37 \mathrm{~mL}$ ) and DMT-dT phosphoramidite (100 $\mathrm{mM}$ in dry acetonitrile, 3 eq. to $\mathrm{OH}$-groups, $0.585 \mathrm{mmol}, 5.85 \mathrm{~mL}$ ). After 60 minutes, $1.0 \mathrm{~mL}$ sample was collected and precipitated twice into cold 2-propanol, washed with 2-propanol, and washed with diethyl ether. After collecting the sample, the reaction mixture contains $0.551 \mathrm{mmol} P^{\prime \prime \prime}$. mCPBA was then added as a powder (2.5 eq. to P'II $1.38 \mathrm{mmol}, 0.340 \mathrm{~g}$ ). After $10 \mathrm{~min}, 1.0 \mathrm{~mL}$ sample was collected and precipitated twice into cold 2-propanol, washed with 2-propanol, and washed with diethyl ether. The remaining reaction mixture $(0.517 \mathrm{mmol}$ DMT-groups) was placed into an ice bath and cooled for 5 minutes before adding DCA $600 \mathrm{mg} / \mathrm{mL}$ in DCM (60 eq. to DMT-groups, $31.0 \mathrm{mmol}, 6.66$ $\mathrm{mL}$ ) and TES (25 eq. to DMT-groups, $12.9 \mathrm{mmol}, 2.06 \mathrm{~mL}$ ). The reaction mixture turned orange, and samples were collected in time intervals by precipitating twice into cold 2-propanol, washing with 2-propanol, and washing with ether. Several samples were collected during the detritylation timescale (the mixture turns colorless after $171 \mathrm{~min}$ ), and several during the depurination timescale (>24 hours). To keep the mixture at exactly $0{ }^{\circ} \mathrm{C}$ overnight, the reaction vessel (50 $\mathrm{mL}$ centrifuge tube with rubber stopper) was placed into a large container with isolating walls filled with ice and de-ionized water.

Note that mCPBA was commercially available as a solid of $\leq 77 \%$, so we added the amount assuming a mCPBAcontent in the powder of $77 \%$. Samples were precipitated twice in all cases, as the $d A(B z)$ phosphoramidite is more difficult to remove than the $\mathrm{dT}$ phosphoramidite.

\section{Synthesis 4-arm PEG- $T_{20}$}

The procedure is described for the first step here (coupling of a T-nucleotide to 4-arm PEG-OH). Vacuum-dried 4-arm PEG-OH (18.914 g, 0.4594 mmol polymer, $1.838 \mathrm{mmol}$ end-groups) was dissolved into ETT solution (250 $\mathrm{mM}$ in dry acetonitrile, 12 eq. to $\mathrm{OH}$-groups, $22.05 \mathrm{mmol}, 88.2 \mathrm{~mL}$ ) and DMT-dT phosphoramidite solution (100 $\mathrm{mM}$ in dry acetonitrile, 3 eq. to $\mathrm{OH}$-groups, $5.51 \mathrm{mmol}, 55.1 \mathrm{~mL}$ ) in a $250 \mathrm{~mL}$ round bottom flask with a large magnetic stirrer bar. After 60 minutes, mCPBA was added as a powder (2.5 eq. to PIII, $13.7 \mathrm{mmol}, 3.070 \mathrm{~g}$ ), leading to a slight warmup of the reaction mixture. After 10 minutes, TCA $600 \mathrm{mg} / \mathrm{mL}$ in DCM (25 eq. to DMTgroups, $134.4 \mathrm{mmol}, 36.6 \mathrm{~mL}$ ) and TES (15 eq. to DMT-groups, $80.6 \mathrm{mmol}, 12.9 \mathrm{~mL}$ ) were added. The reaction

mixture turned to an intense orange color, which slowly disappeared over the course of 10 minutes indicating complete detritylation and scavenging of the DMT-cation. The reaction mixture was precipitated slowly into a 15fold volume (in this step $3 \mathrm{~L}$ ) $-30{ }^{\circ} \mathrm{C} 2$-propanol under vigorous stirring with an overhead stirring setup. After precipitation, the polymer was isolated by vacuum filtration through a Macherey-Nagel 1670 filter paper, washed with $1 \mathrm{~L}$ of room temperature 2-propanol, and washed with $0.5 \mathrm{~L}$ of room temperature diethyl ether. The product was filled into a pre-weighed flask with a magnetic stirrer bar, pre-dried on high vacuum for 1-2 hours to remove 
most of the solvents, and then dried overnight at a vacuum of 0.001 mbar. The product was isolated as a white powder (19.3 g, $0.454 \mathrm{mmol}, 98.8 \%$ recovery). After each step, samples were removed for analysis by ${ }^{1} \mathrm{H} /{ }^{31} \mathrm{P}$ NMR, HPLC, AGE, and SEC. Note that mCPBA was commercially available as a solid of $\leq 77 \%$, so we added the amount assuming a mCPBA-content in the powder of $77 \%$.

\section{Further comments on 4-arm PEG- $\mathrm{T}_{20}$ synthesis}

The synthesis proceeded uneventful during the first 5 one-pot dT addition steps. From 5 steps onwards, the material started to take on a more fiber-like form upon precipitation into $-30^{\circ} \mathrm{C} 2$-propanol leading to decreased purification efficiency and difficult drying of the fiber aggregates. To overcome this, the temperature of the 2propanol used for precipitation was increased stepwise until room temperature at step 9. At this point, additional dilution with DCM after the detritylation completed was required to obtain a well-dispersed precipitate, and the volume of 2-propanol was increased proportionally to maintain a minimal 15-fold volume of non-solvent. From step 9 onwards, dissolving the polymer in the acetonitrile mixture with phosphoramidite and ETT became increasingly slow, and additional dry acetonitrile was added increasing with each step. From 11 steps onwards, the precipitate partially consisted of rather large aggregates that would be difficult to dry on vacuum. Therefore, the precipitate was re-dissolved into 95/5 acetonitrile/DMSO and precipitate into a 20 -fold volume of diethyl ether. This leads to small precipitate particles which can be efficiently dried on high vacuum overnight. Due to the highly aggregating nature of the precipitate, it became possible to avoid the first filtration and collect the precipitate by detaching it from the stainless steel propeller stirrer directly. We furthermore observed that after 13 steps, the coupling solution would be somewhat grey/turbid due to decreased solubility, but would turn almost clear several minutes after dissolving, possibly because the coupled DMT-on product is more soluble. During the last 3 coupling steps $(18,19,20)$, the reaction mixture was turbid even after dilution with anhydrous acetonitrile and of gel-like consistency. After completing the final step (20), the product is re-precipitated into diethyl ether from 50/50 DMSO/acetonitrile rather than 5/95, resulting in a white powder. In two instances (after step 6 and step 10), the material contained a detectable amount of $\mathrm{H}$-phosphonate seen by a double peak at $\sim 8 \mathrm{ppm}$ in ${ }^{31} \mathrm{P}-\mathrm{NMR}$ (the activated phosphoramidite reacts rapidly to $\mathrm{H}$-phosphonate with trace water). In this case, the product was re-dissolved $10 \%$ in acetonitrile and precipitated, filtered, and washed again.

\section{Synthesis 4-arm PEG-A20}

The procedure is described for the first step here (coupling of an A-nucleotide to 4-arm PEG-OH). Vacuum-dried 4-arm PEG-OH (10.097 g, 0.2453 mmol polymer, 0.981 mmol end-groups) was dissolved into ETT solution (250 $\mathrm{mM}$ in dry acetonitrile, 12 eq. to $\mathrm{OH}$-groups, $11.8 \mathrm{mmol}, 47.1 \mathrm{~mL}$ ) and DMT-dA(Bz) phosphoramidite solution (100 mM in dry acetonitrile, 3 eq. to OH-groups, $2.94 \mathrm{mmol}, 29.4 \mathrm{~mL}$ ) in a $250 \mathrm{~mL}$ round bottom flask with a large magnetic stirrer bar. After 60 minutes, mCPBA was added as a powder (2.5 eq. to PIII, $7.36 \mathrm{mmol}, 1.814$ 
$\mathrm{g}$ ), leading to a slight warmup of the reaction mixture. After 5 minutes, the reaction mixture was placed into an ice bath, and stirred for 5 more minutes. Then, DCA $600 \mathrm{mg} / \mathrm{mL}$ in DCM (70 eq. to DMT-groups, $205.4 \mathrm{mmol}$, $44.1 \mathrm{~mL}$ ) and TES (25 eq. to DMT-groups, $73.3 \mathrm{mmol}, 11.7 \mathrm{~mL}$ ) were added. This turned the reaction mixture to an intense orange color, which slowly disappeared over the course of 110 minutes indicating complete detritylation and scavenging of the DMT-cation. The reaction mixture was then precipitated into a minimum 15fold volume (in this step $2 \mathrm{~L}$ ) $-30^{\circ} \mathrm{C}$ 2-propanol under vigorous stirring with an overhead stirring setup. After precipitation, the polymer was isolated by vacuum filtration through a Macherey-Nagel 1670 filter paper, and washed with $0.5 \mathrm{~L}$ of room temperature 2-propanol. The polymer was then re-dissolved in DCM as a 10 wt\% solution, and precipitated again into a 15 -fold excess $-30^{\circ} \mathrm{C} 2$-propanol under vigorous stirring. The product was isolated by filtration again, washed with $0.3 \mathrm{~L}$ room temperature 2-propanol, washed with $0.4 \mathrm{~L}$ room temperature diethyl ether, and filled into a pre-weighed round-bottom flask with a magnetic stirrer bar. The product was predried on high vacuum with a liquid nitrogen trap for 1-2 hours to remove most of the solvents, and then dried overnight without a liquid nitrogen trap with a vacuum of $0.001 \mathrm{mbar}$. The product was isolated as a white powder (10.0 g, $0.232 \mathrm{mmol}, 94.9 \%$ recovery). After each step, samples were removed for analysis by ${ }^{1} \mathrm{H} /{ }^{31} \mathrm{P}-\mathrm{NMR}$, HPLC, AGE, and SEC. Note that mCPBA was commercially available as a solid of $\leq 77 \%$, so we added the amount assuming a mCPBA-content in the powder of $77 \%$.

\section{Comments on 4-arm PEG-A20 synthesis}

The synthesis of 4-arm PEG-A 20 proceeded similar to that of 4-arm PEG-T20, with solubility and precipitation becoming more difficult as the synthesis proceeded. The main difference is that in addition to the precipitation after the one-pot nucleotide addition, an additional re-precipitation from DCM into 2-propanol was required to remove all residual phosphoramidite. This is the result of the much larger size of the Bz-prodected $\mathrm{dA}$ phosphoramidite compared to dT. Up to step 13, the fiber-like precipitate could be mechanically dispersed with a stainless steel overhead propeller stirrer to enable efficient drying. From step 13 onwards, an additional precipitation from DCM + 5 vol\% DMSO into diethyl ether was required to facilitate drying the material. To save time, the precipitate did not have to be filtered anymore during the precipitations into 2-propanol, but could instead be collected directly from the stirrer in the form of strongly aggregating fibers. The re-precipitation into 2propanol was done from a DMSO solution rather than DCM solution from step 15 onwards to facilitate better purification at the expense of recovery yield. As opposed to the synthesis of 4-arm PEG-T 20 , the reaction mixture stayed mostly clear during the coupling steps even up to 20 bases provided the mixture was diluted with anhydrous acetonitrile accordingly. The solubility of the oligo-A arms in acetonitrile seems better compared to oligo-T. In 3 instances (after step 13, step 14, and step 16) the material contained a detectable amount of $\mathrm{H}$ phosphonate seen by a double peak at $\sim 8$ ppm in ${ }^{31} \mathrm{P}-\mathrm{NMR}$. In this case, the product was re-dissolved $10 \mathrm{wt} \%$ in DCM (steps 13 and 14) or DMSO (step 16) and precipitated, filtered, and washed again. 


\section{Preparation of model network hydrogel from 4-arm PEG-T 20 and 4-arm PEG-A $A_{20}$}

First, the 4-arm PEG-T 20 (with cyanoethyl protecting group) and 4-arm PEG-A 20 (with cyanoethyl and benzoyl protecting groups) were deprotected in concentrated ammonia/methylamine (AMA) solution for 30 min and 120 min respectively at a concentration of $50 \mathrm{mg} / \mathrm{mL}$. The solutions were then diluted 10-fold with milliQ water, and dried by lyophilization. The deprotected products were dissolved at $100 \mathrm{mg} / \mathrm{mL}$ in phosphate buffer $(\mathrm{pH} 8.0,75$ mM phosphate, $1 \mathrm{mM}$ EDTA, $150 \mathrm{mM} \mathrm{Na}^{+}$), after which the concentration was measured by UV-Vis (taking into account the presence of oligo-T/A impurities which were quantified by HPLC) and adjusted to $0.90 \mathrm{mM}$ PEG$\mathrm{T} / \mathrm{A}_{20}$ with phosphate buffer. Both solutions were heated to $60{ }^{\circ} \mathrm{C}$, mixed in equal volumes, and cooled down slowly to room temperature. The gel was then heated back up to $65^{\circ} \mathrm{C}$ when placed into the rheometer right before the top plate was moved in place. 


\section{Supplementary analysis}

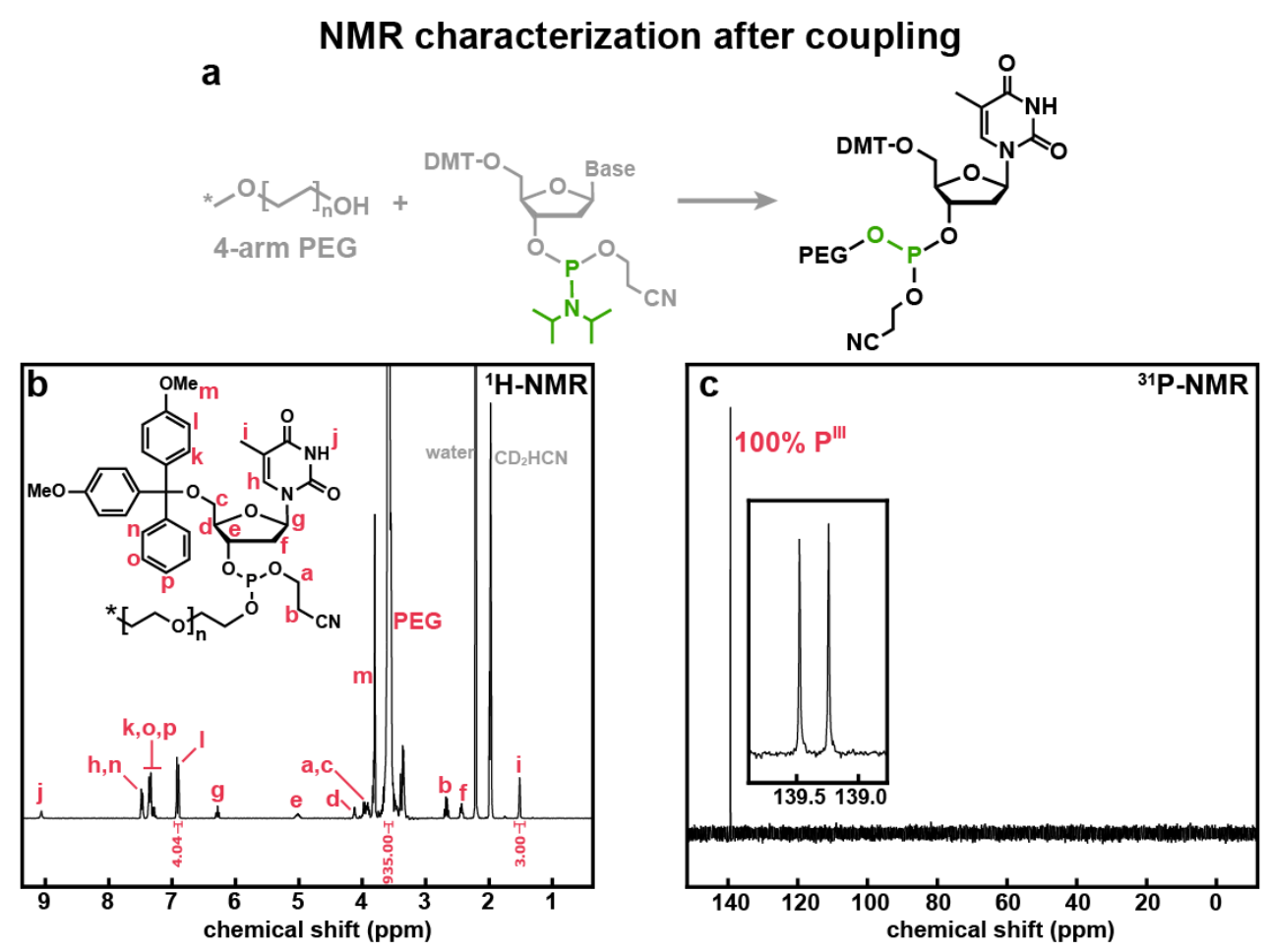

Figure S1. (a) Reaction scheme for the coupling of 4-arm PEG-OH with DMT-dT phosphoramidite resulting in 4-arm PEG-dT-DMT phosphite triester (PIII). (b) ${ }^{1} \mathrm{H}-\mathrm{NMR}\left(\mathrm{CD}_{3} \mathrm{CN}\right)$ of 4-arm PEG ${ }_{41 \mathrm{k}}$-dT-DMT (P'II) with integrals for the peaks that are compared to the PEG peak $(935 \mathrm{H})$ to quantify coupling (methyl $3 \mathrm{H}$ ) and degree of DMTon (DMT 4H). (b) Corresponding ${ }^{31} \mathrm{P}-\mathrm{NMR}$ showing only phosphite triester ( $\left.\mathrm{P}^{\prime \prime \prime}\right)$, with an inset showing the split peaks due to the presence of two diastereomers. * symbolizes the core of the 4-arm PEG. 
NMR characterization after oxidation

a

a
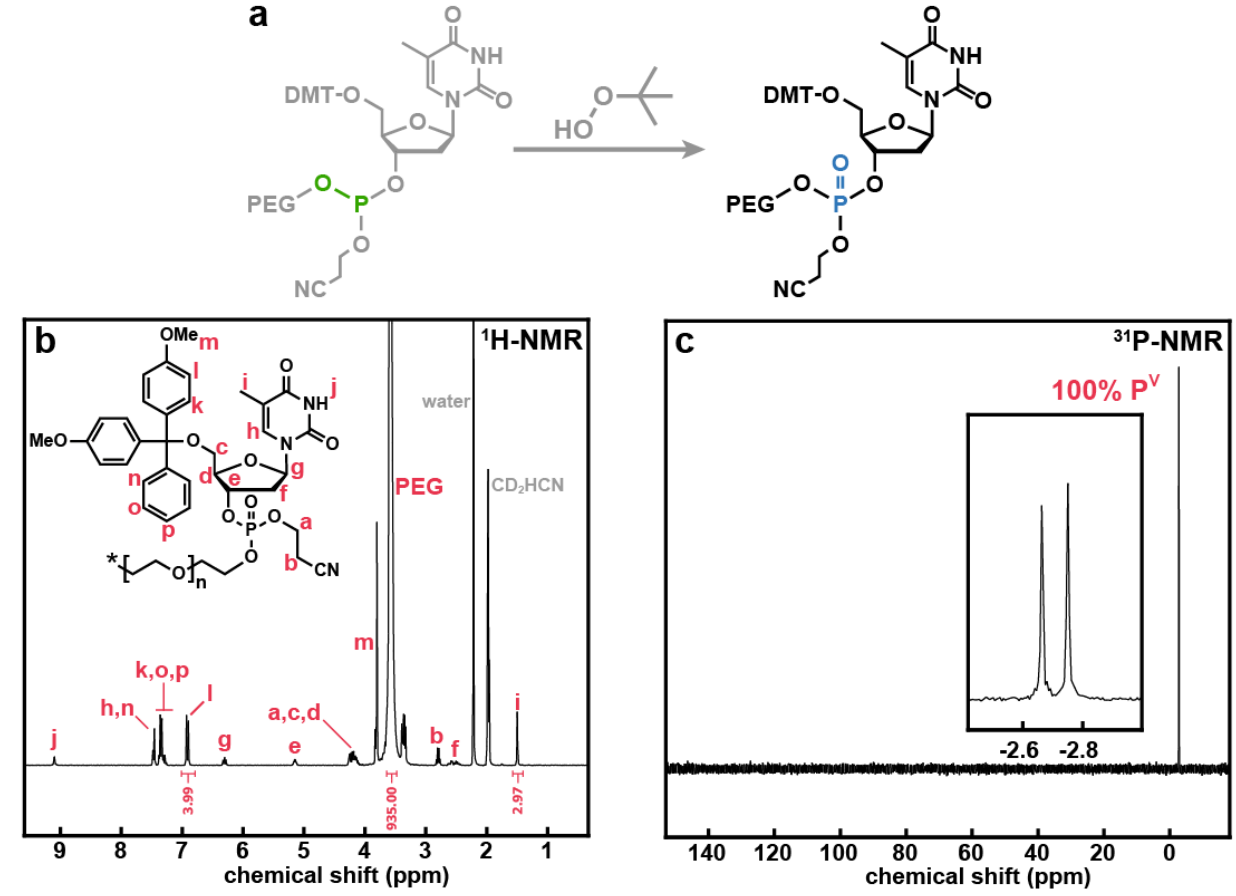

Figure S2. (a) Reaction scheme for the oxidation of 4-arm PEG-dT-DMT phosphite triester (PIII) with anhydrous TBHP yielding 4-arm PEG-dT-DMT phosphate triester $\left(P^{V}\right)$. (b) ${ }^{1} \mathrm{H}-\mathrm{NMR}\left(\mathrm{CD}_{3} \mathrm{CN}\right)$ of 4-arm PEG $41 \mathrm{k}^{-\mathrm{dT}-\mathrm{DMT}}\left(\mathrm{P}^{\mathrm{V}}\right)$ with integrals for the peaks that are compared to the PEG peak $(935 \mathrm{H})$ to quantify coupling (methyl $3 \mathrm{H})$ and degree of DMT-on (DMT 4H). (b) Corresponding ${ }^{31} \mathrm{P}-\mathrm{NMR}$ showing only phosphate triester $\left(\mathrm{P}^{\mathrm{V}}\right)$, with an inset showing the split peaks due to the presence of two diastereomers. * symbolizes the core of the 4-arm PEG. 


\section{NMR characterization after detritylation}

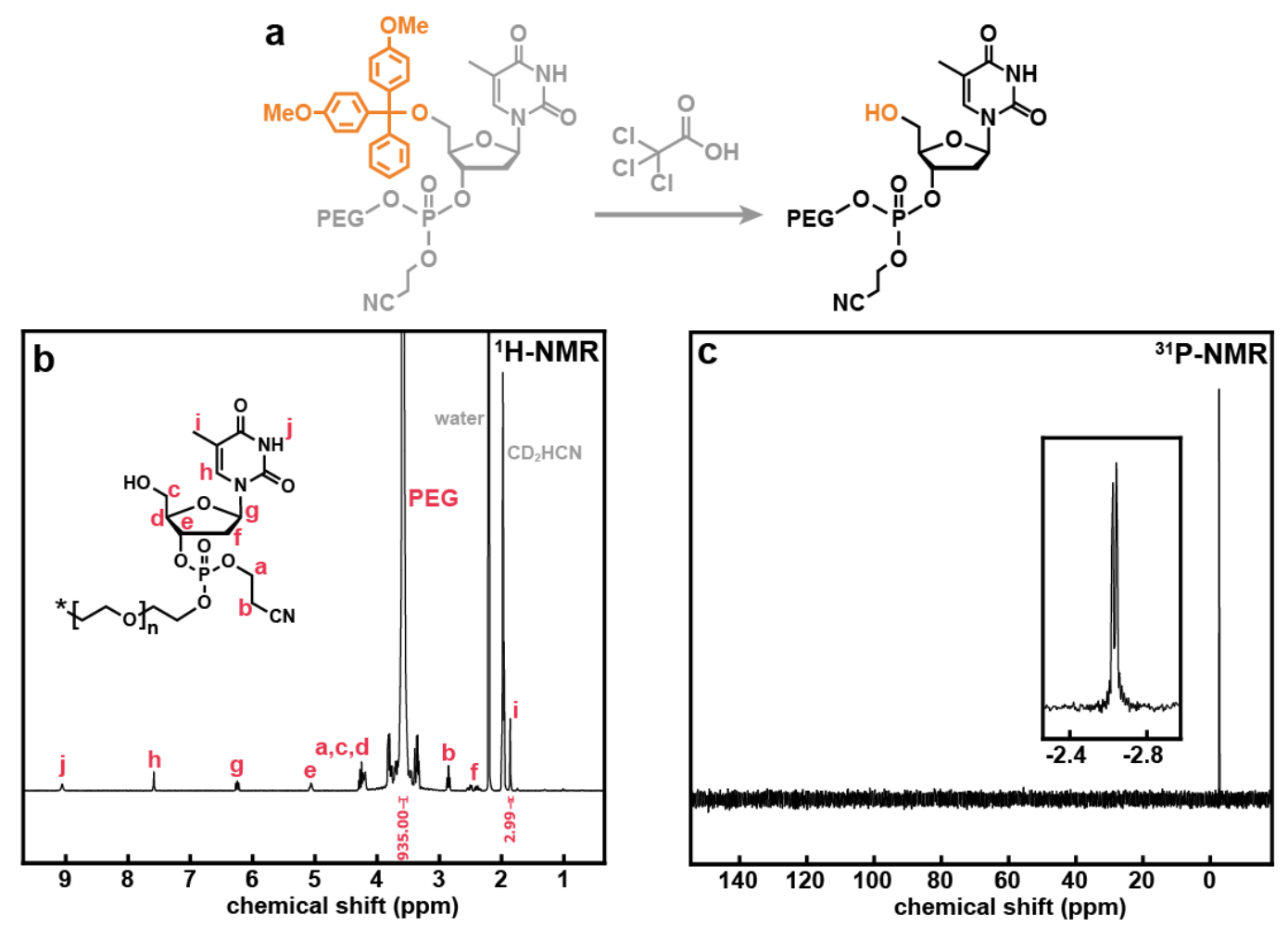

Figure S3. (a) Reaction scheme for the detritylation of 4-arm PEG-dT-DMT phosphate triester (PV) with TCA

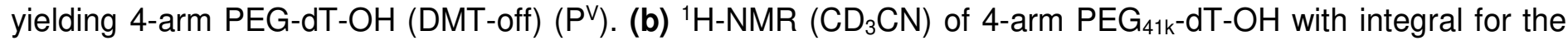
peak that is compared to the PEG peak $(935 \mathrm{H})$ to quantify coupling (methyl $3 \mathrm{H})$. (c) Corresponding ${ }^{31} \mathrm{P}-\mathrm{NMR}$ showing only phosphate triester $\left(\mathrm{P}^{\mathrm{V}}\right)$, with an inset showing the split peaks due to the presence of two diastereomers. 


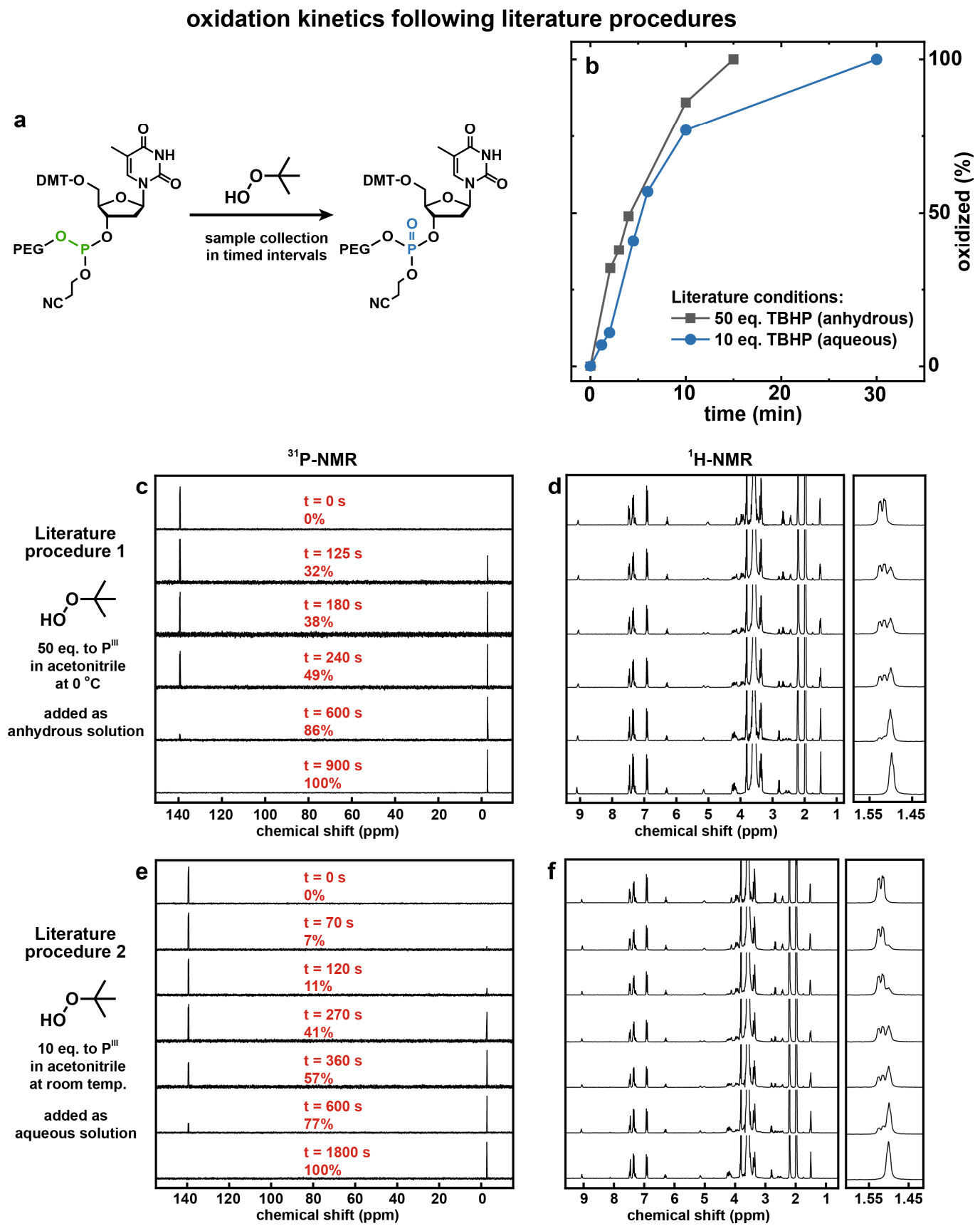

Figure S4. Oxidation kinetics of 4-arm PEG-dT-DMT (PIII) following literature conditions. (a) Reaction scheme showing the oxidation of the phosphite to phosphate with THBP following two different literature procedures: (1) PEG-dT-DMT (PIII) $5 \mathrm{wt} \%$ in acetonitrile with 50 eq. (to $\mathrm{P}^{\prime \prime \prime}$ ) added as $5.5 \mathrm{M}$ in decane (anhydrous), (2) PEG-dT-DMT (PIII) 10 wt\% in acetonitrile with 10 eq. (to PIII) added as $70 \%$ in water (aqueous). (b) Kinetics of oxidation following the two different literature conditions. (c) ${ }^{31} \mathrm{P}-\mathrm{NMR}$ and (d) ${ }^{1} \mathrm{H}-\mathrm{NMR}\left(\mathrm{CD}_{3} \mathrm{CN}\right)$ for the oxidation with 50 eq. anhydrous TBHP. (e) ${ }^{31} \mathrm{P}-\mathrm{NMR}$ and (f) ${ }^{1} \mathrm{H}-\mathrm{NMR}\left(\mathrm{CD}_{3} \mathrm{CN}\right)$ for the oxidation with 10 eq. aqueous TBHP. Degree of oxidation is determined as the ratio of $\mathrm{P}^{\mathrm{III}}$ and $\mathrm{PV}^{\mathrm{V}}$ integrals. Although $\mathrm{H}$-decoupled ${ }^{31} \mathrm{P}$-NMR is not strictly quantitative, neither product has an $\mathrm{H}$-nucleus in $\alpha$ - or $\beta$-position to $\mathrm{P}$, and the oxidation ratio could be verified by comparing some peaks in ${ }^{1} \mathrm{H}-\mathrm{NMR}$. 


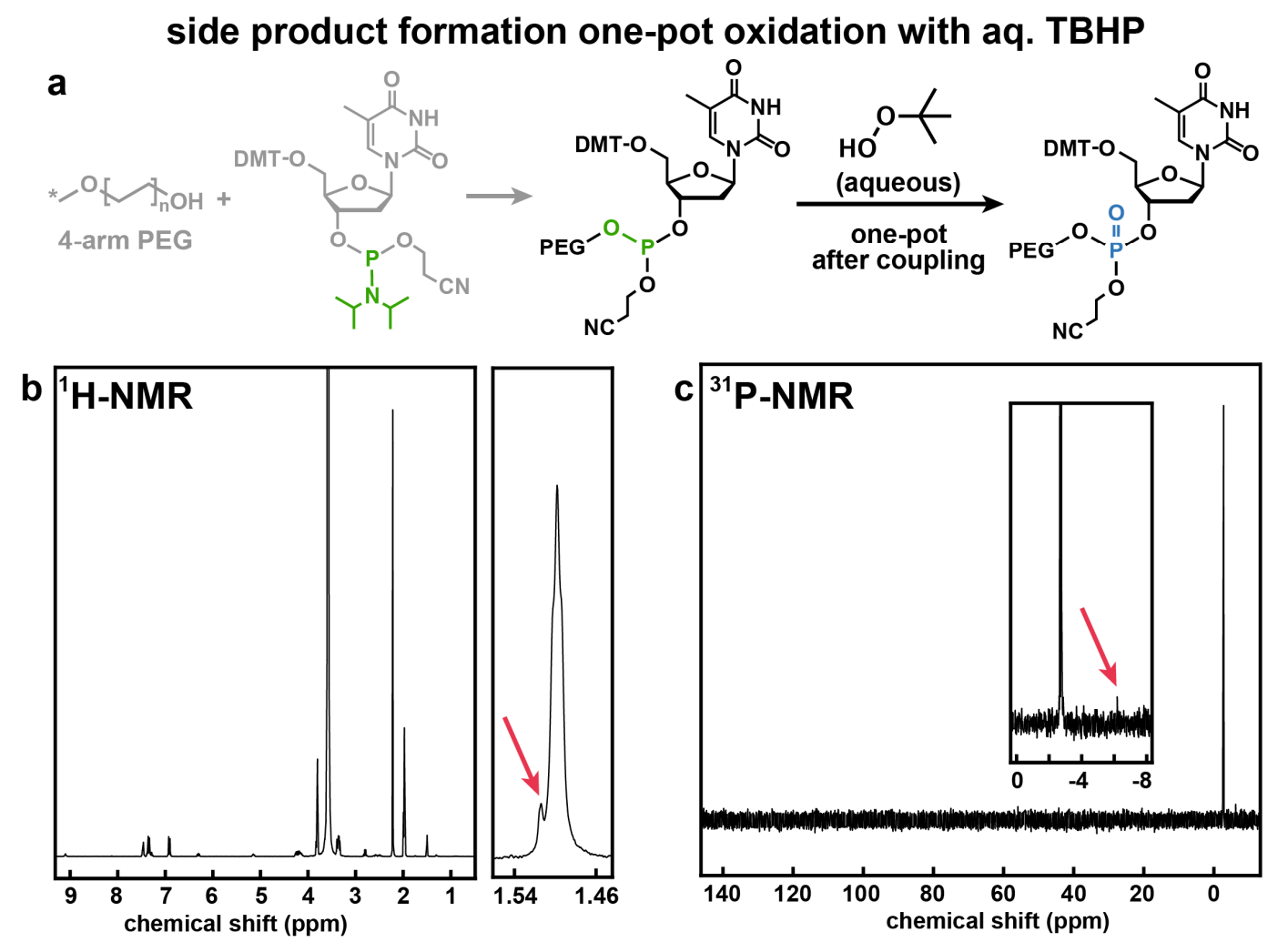

Figure S5. (a) Reaction scheme showing the coupling of DMT-dT phosphoramidite with 4-arm PEG-OH in acetonitrile followed by the one-pot oxidation by adding 10 eq. (to $\mathrm{P}^{\mathrm{III}}$ ) of TBHP as an aqueous solution (70\% TBHP in water). (b) ${ }^{1} \mathrm{H}-\mathrm{NMR}$ spectrum $\left(\mathrm{CD}_{3} \mathrm{CN}\right)$ of 4 -arm $\mathrm{PEG} \mathrm{G}_{4 \mathrm{k}}$-dT-DMT $\left(\mathrm{P}^{\mathrm{V}}\right)$ oxidized one-pot with 10 equivalents (to $\mathrm{P}^{\mathrm{III}}$ ) of aqueous TBHP, with a detailed view of a side product peak next to the dT-methyl $3 \mathrm{H}$ signal. (b) Corresponding ${ }^{31} \mathrm{P}-\mathrm{NMR}$ spectrum $\left(\mathrm{CD}_{3} \mathrm{CN}\right)$. 


\section{oxidation kinetics for one-pot oxidation after coupling}
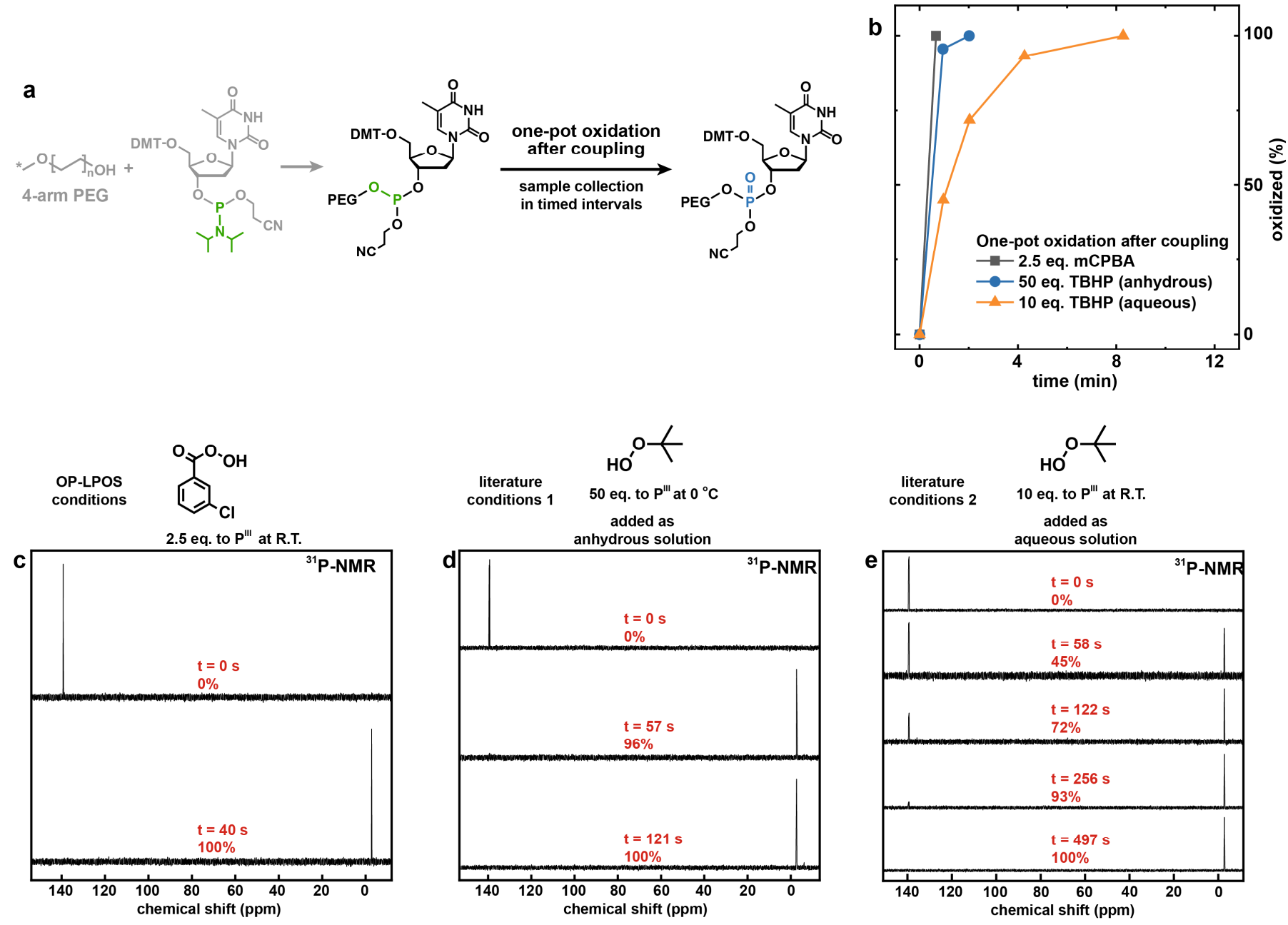

Figure S6. (a) Reaction scheme showing the one-pot oxidation of the phosphite to phosphate after the coupling step. (b) Kinetic plot for the one-pot oxidation with three different conditions: (1) mCPBA 2.5 equivalents to PIII at room temperature, (2) TBHP 50 equivalents to $\mathrm{P}^{\mathrm{Ill}}$ added as an anhydrous solution in decane at $0{ }^{\circ} \mathrm{C}$, (3) TBHP 10 equivalents to PIII added as a solution in water at room temperature. (c-e) ${ }^{31} \mathrm{P}-\mathrm{NMR}\left(\mathrm{CD}_{3} \mathrm{CN}\right)$ used to determine the degree of oxidation by comparing integrals of the phosphite triester ( $\left.P^{\prime \prime \prime}\right)$ and phosphate triester $\left(P^{V}\right)$ peaks. Degree of oxidation is determined as the ratio of $P^{I I I}$ and $P^{V}$ integrals. Although $\mathrm{H}$-decoupled ${ }^{31} \mathrm{P}$ NMR is not strictly quantitative, neither product has an $\mathrm{H}$-nucleus in $\alpha$ - or $\beta$-position to $\mathrm{P}$, and the oxidation ratio could be verified by comparing peaks in ${ }^{1} \mathrm{H}-\mathrm{NMR}$. Only mCPBA leads to a clean oxidation. 

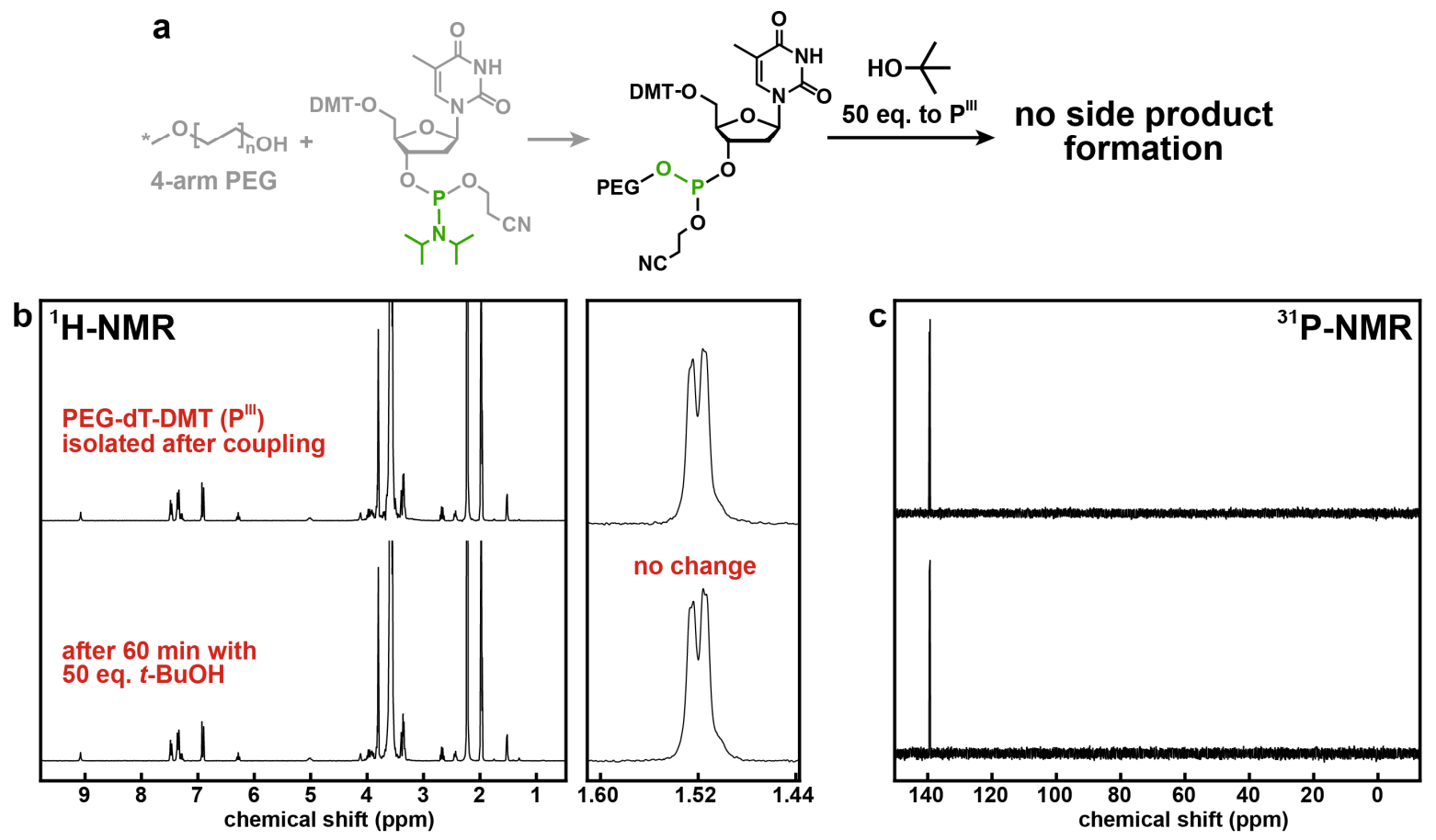

Figure S7. (a) Reaction scheme for an experiment to investigate if the side product observed during one-pot

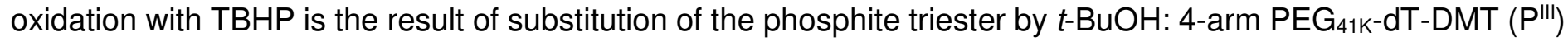
(b) ${ }^{1} \mathrm{H}$-NMR spectrum $\left(\mathrm{CD}_{3} \mathrm{CN}\right)$ of 4 -arm PEG ${ }_{41 \mathrm{k}}$-dT-DMT (PIII) before and after stirring with 50 equivalents (to $\mathrm{P}^{\mathrm{III}}$ ) of $t-\mathrm{BuOH}$ for 60 minutes, with an inset showing the lack of any side product formation. (c) Corresponding ${ }^{31} \mathrm{P}-\mathrm{NMR}$ spectrum $\left(\mathrm{CD}_{3} \mathrm{CN}\right)$. The side product of Figure $\mathrm{S} 7$ does not form only based on addition of t-BuOH, but is a result of the presence of other reagents (such as TBHP) or a combination of reagents. 
a

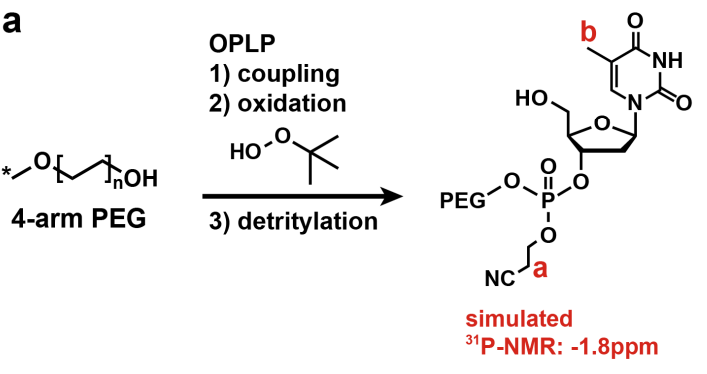

3 eq phosphoramidite

'H-NMR

C $\mathrm{dT}: \mathbf{9 7 . 7 \%}$ CE: $97.5 \%$ tBu (1): $2.2 \%$

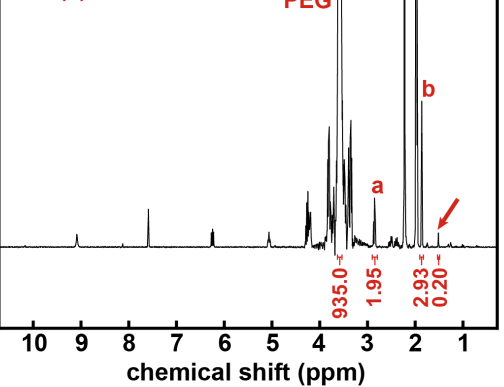

4 eq. phosphoramidite

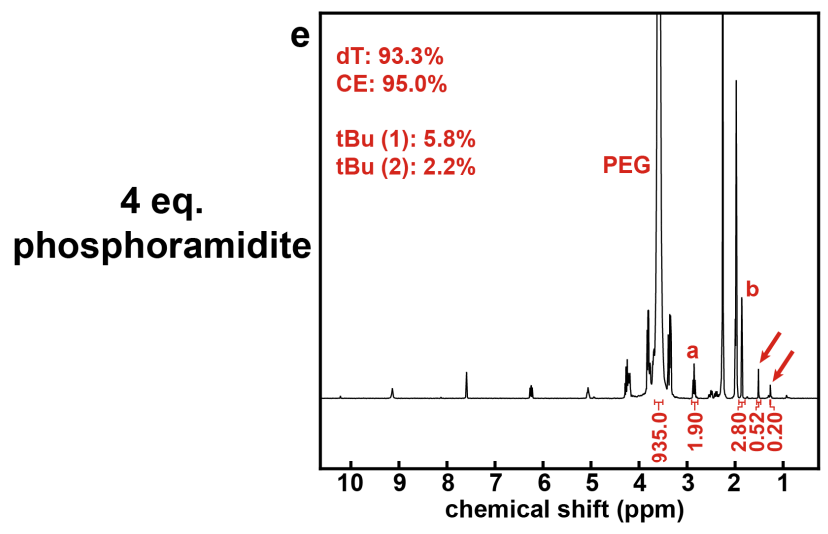

tBu (2): $2.2 \%$ phosphoramidite

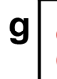

gT: $91.3 \%$ CE: $96.0 \%$ tBu (1): $7.7 \%$ tBu (2): $3.2 \%$

$6 \mathrm{eq}$

\section{phosphoramidite} (

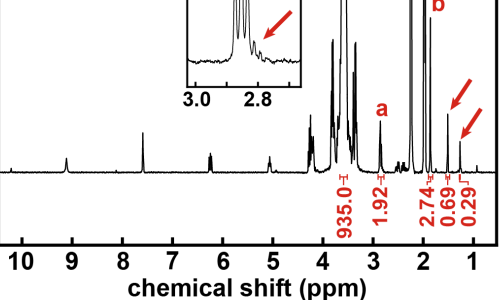

possible side products
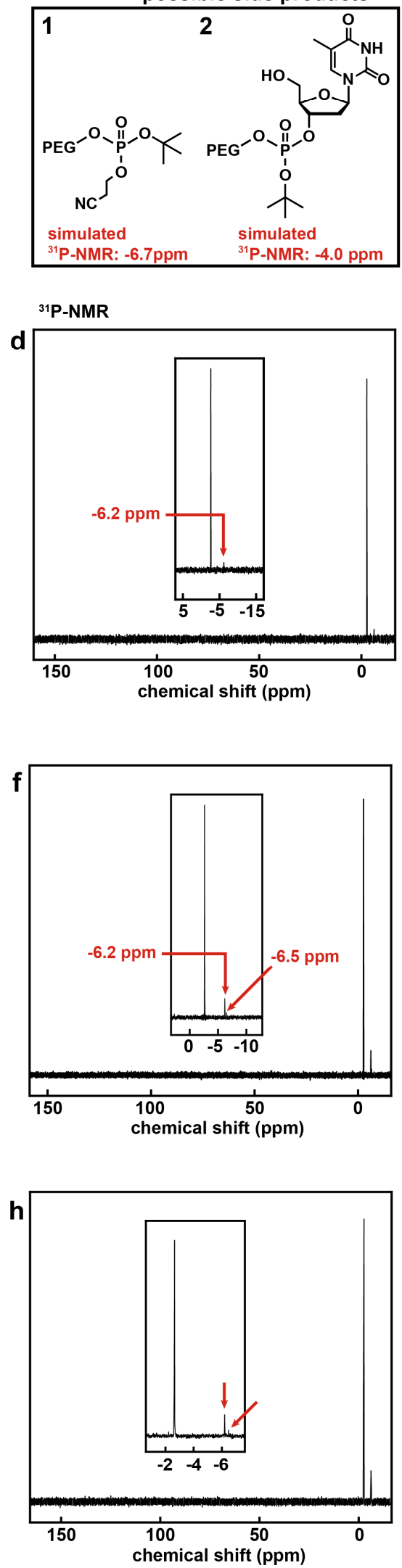

Figure S8. Analysis of the side reactions during one-pot oxidation with TBHP. (a) Reaction scheme for the coupling and oxidation with TBHP followed by detritylation in a one-pot fashion, with (b) the structures of 
suggested side products. (c-h) ${ }^{1} \mathrm{H}-\mathrm{NMR}\left(\mathrm{CD}_{3} \mathrm{CN}\right)$ and ${ }^{31} \mathrm{P}-\mathrm{NMR}$ of 4-arm $\mathrm{PEG}_{41 \mathrm{~K}}$-dT (DMT-off) synthesized with 3 , 4, and 6 eq. phosphoramidite to $\mathrm{OH}$-groups. Insets and arrows highlight side product peaks. The amount of dT (peak b, 3H), CE-groups (peak a, $2 \mathrm{H}$ ), and tBuO-substitution products $(9 \mathrm{H})$ per arm is determined by comparing the integrals to the PEG peak $(935 \mathrm{H})$. Coupling is conducted with 3,4 , and 6 eq. dT phosphoramidite to $\mathrm{OH}$-groups and 4 eq. ETT to phosphoramidite for 60 minutes, one-pot oxidation with 50 eq. TBHP to phosphoramidite for 30 minutes, and one-pot detritylation with TCA/TES $25 / 15$ to DMT groups. The products are isolated by precipitating the one-pot reaction mixture into $-30{ }^{\circ} \mathrm{C} 2$-propanol followed by re-precipitation from DCM into - $30{ }^{\circ} \mathrm{C} 2$-propanol.
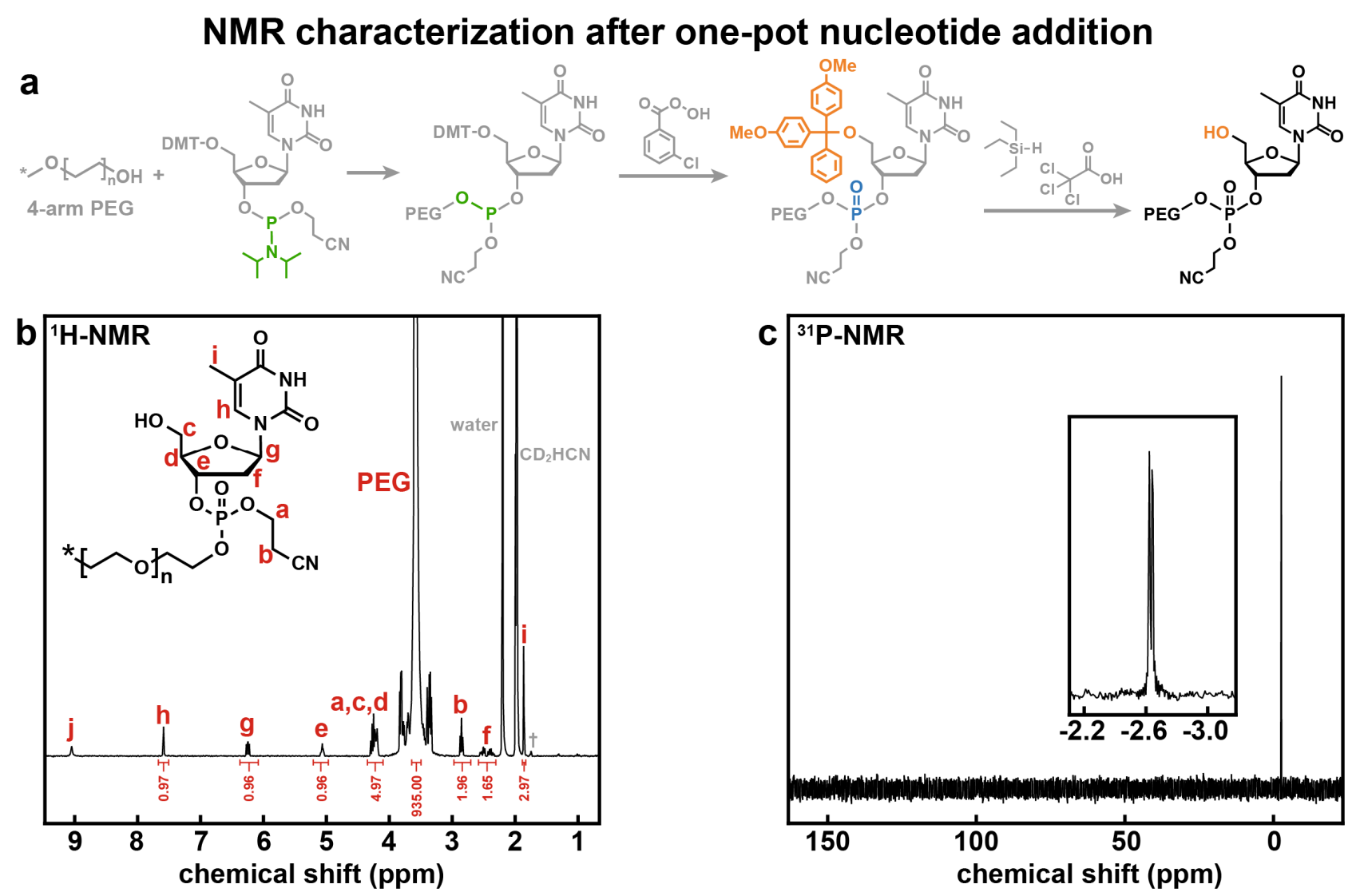

Figure S9. (a) Reaction scheme for the synthesis of 4-arm $P E_{41 K}-d T$ in a one-pot process starting from 4-arm PEG-OH. (b) ${ }^{1} \mathrm{H}-\mathrm{NMR}$ spectrum $\left(\mathrm{CD}_{3} \mathrm{CN}\right)$ of 4-arm PEG ${ }_{41 \mathrm{~K}-\mathrm{dT}}$ (DMT-off) synthesized by one-pot nucleotide addition, and isolated by precipitating into $-30^{\circ} \mathrm{C} 2$-propanol, filtration, then washing with 2-propanol. + indicates the ${ }^{13} \mathrm{C}$ satellite peak of the residual solvent peak $\left(\mathrm{CD}_{2} \mathrm{HCN}\right)$. (c) Corresponding ${ }^{31} \mathrm{P}-\mathrm{NMR}$ spectrum with an inset showing the phosphate triester peak in detail. 

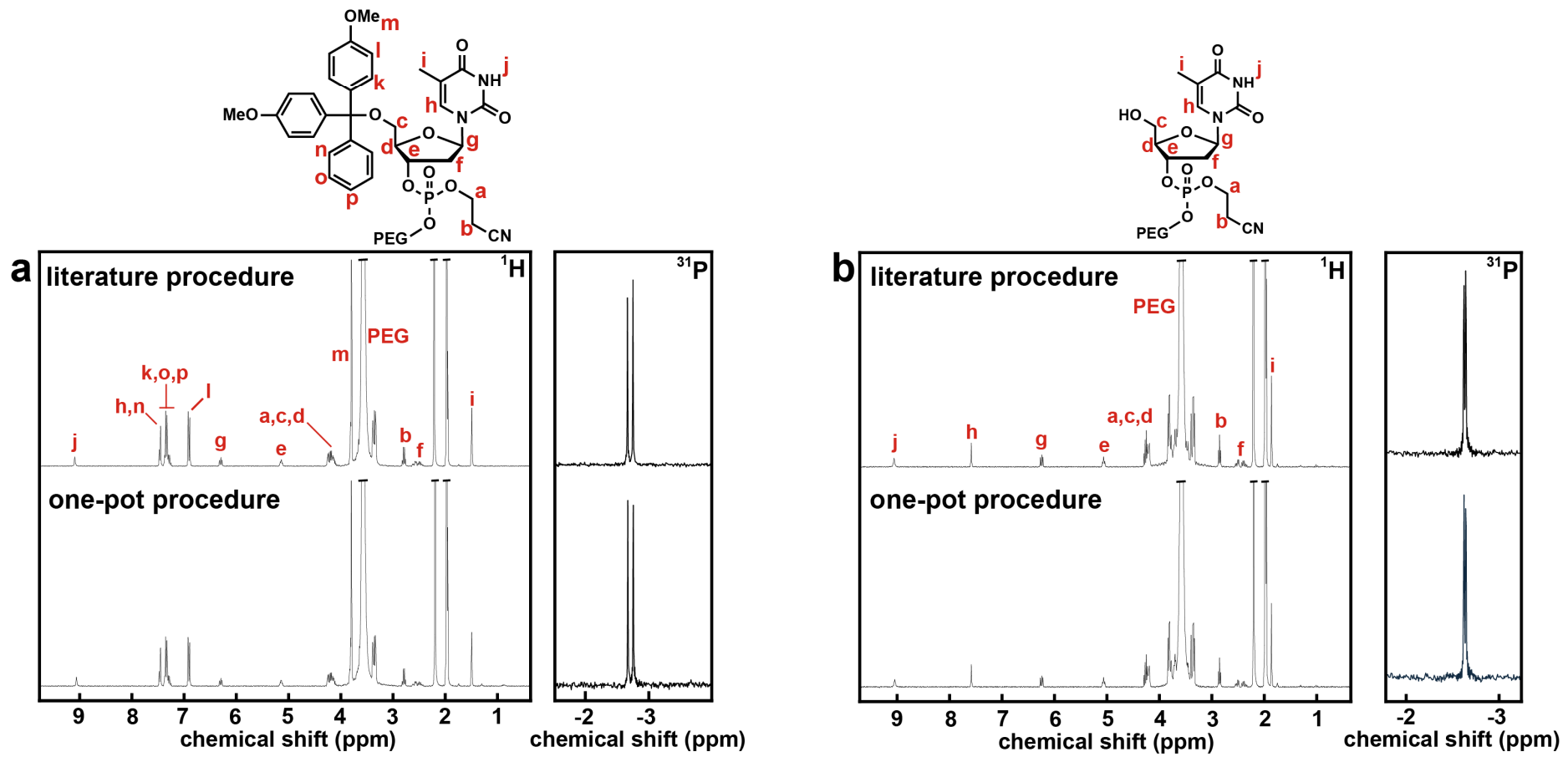

Figure S10. Comparison of ${ }^{1} \mathrm{H}-\mathrm{NMR}\left(\mathrm{CD}_{3} \mathrm{CN}\right)$ and corresponding ${ }^{31} \mathrm{P}-\mathrm{NMR}$ for (a) 4-arm PEG-dT-DMT $\left(\mathrm{PV}^{\mathrm{V}}\right)$ and (b) 4-arm PEG-dT-OH $\left(P^{\vee}\right)$, showing no difference between the respective products obtained by the sequential literature procedure (intermediate workup after coupling, oxidation, detritylation $(2 x)$ ) or one-pot liquid-phase oligonucleotide synthesis. 


\section{removal of reagents by precipitation in diethyl ether}

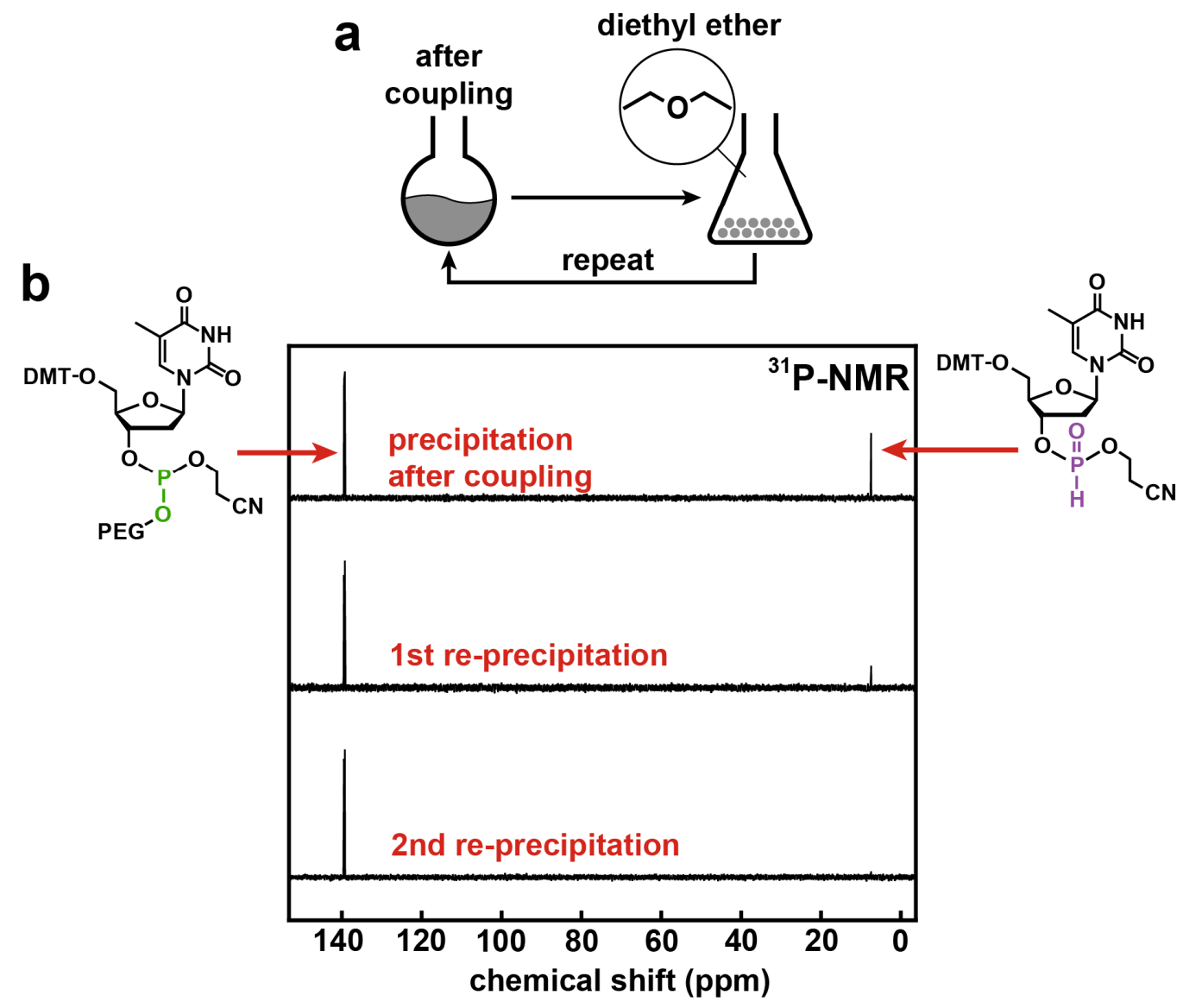

Figure S11. (a) The removal of excess phosphoramidite following current literature procedure takes place after coupling by repeated precipitation into diethyl ether. (b) ${ }^{31} \mathrm{P}-\mathrm{NMR}\left(\mathrm{CD}_{3} \mathrm{CN}\right)$ of 4-arm PEG-dT-DMT (PIII) after several precipitation into diethyl ether followed by washing with diethyl ether. The initial precipitation is from the reaction mixture in acetonitrile, and re-precipitations are done from $10 \mathrm{wt} \%$ in DCM. The phosphoramidite is not observed in ${ }^{31} \mathrm{P}-\mathrm{NMR}$ as it reacts with trace water to form the $\mathrm{H}$-phosphonate in the presence of ETT activator. Note that the ratio of integrals does not reflect the relative amounts of phosphite triester and $\mathrm{H}$-phosphonate due to $\mathrm{H}$-decoupling in the ${ }^{31} \mathrm{P}-\mathrm{NMR}$ and the presence of $\mathrm{H}$ in $\alpha$-position to $\mathrm{P}$ in the $\mathrm{H}$-phosphonate. Diethyl ether is not a good precipitation solvent for removing reagents from the product. 

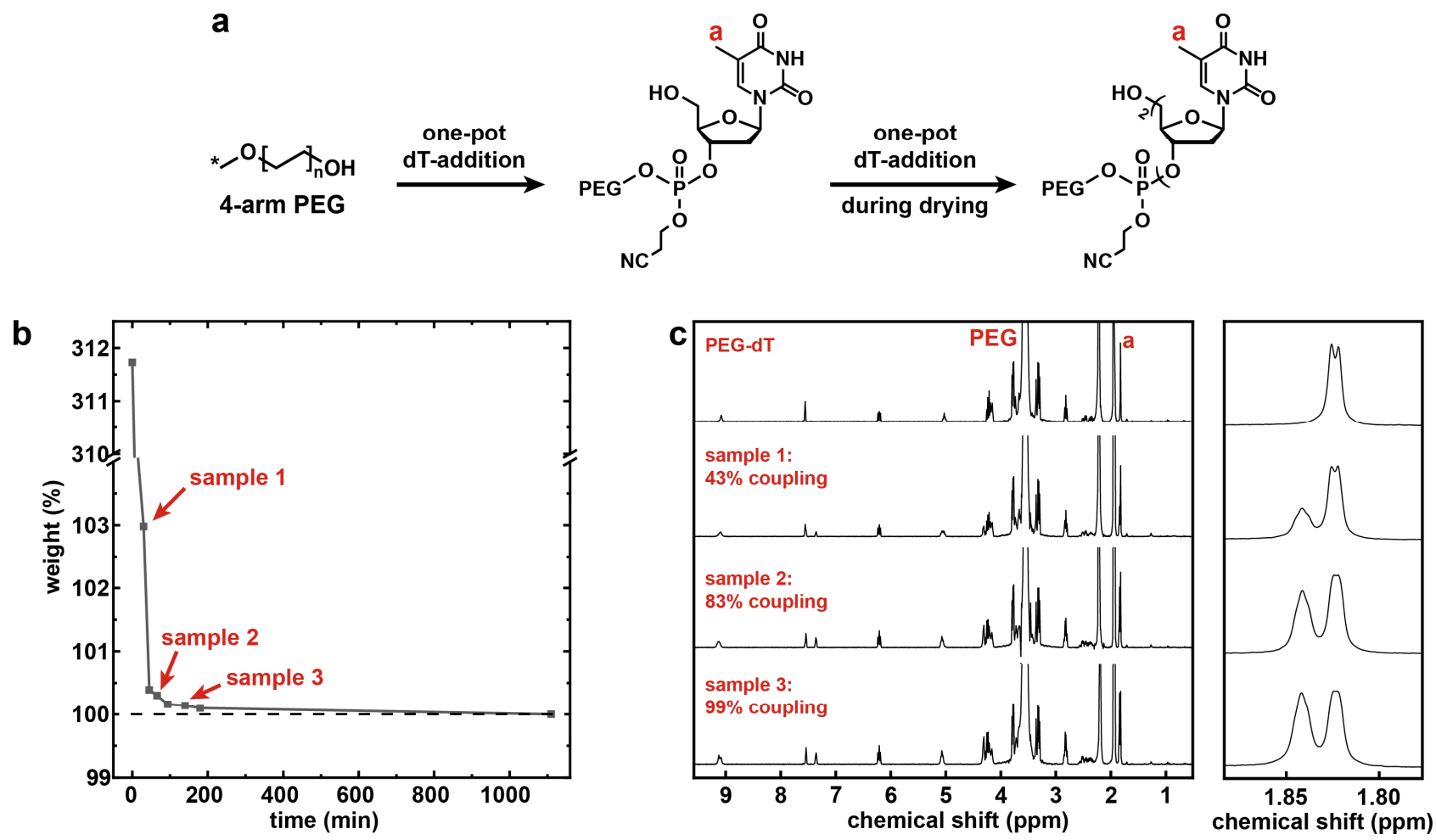

Figure S12. Correlation of drying time to material weight and coupling efficiency of a second nucleotide. (a) A dT-nucleotide is coupled to $2 \mathrm{~g}$ PEG-OH in a one-pot fashion, and during the drying of this material samples are collected and coupled a second time. (b) Weight decrease during the drying process under vacuum. Weight is measured relative to the product dried overnight. (c) ${ }^{1} \mathrm{H}-\mathrm{NMR}\left(\mathrm{CD}_{3} \mathrm{CN}\right)$ of PEG-dT and the samples coupled during the drying process. The coupling efficiency was determined by comparing the integral of the PEG arm $(935 \mathrm{H})$ to the thymine methyl group ( $3 \mathrm{H}$ per nucleotide). Conditions: coupling with 3 eq. dT phosphoramidite and 12 eq. ETT to $\mathrm{OH}$-groups for 60 minutes, oxidation with 2.5 eq. mCPBA to phosphoramidite for 10 minutes, detritylation with 25/15 eq. TCA/TES to DMT groups. Precipitation of $2 \mathrm{~g}$ PEG-dT into $400 \mathrm{~mL}-30^{\circ} \mathrm{C} 2$-propanol, washed with $200 \mathrm{~mL}$ RT 2-propanol and $300 \mathrm{~mL}$ room temperature diethyl ether after filtration. 
NMR characterization of intermediate samples during 4-arm PEG-T ${ }_{20}$ synthesis
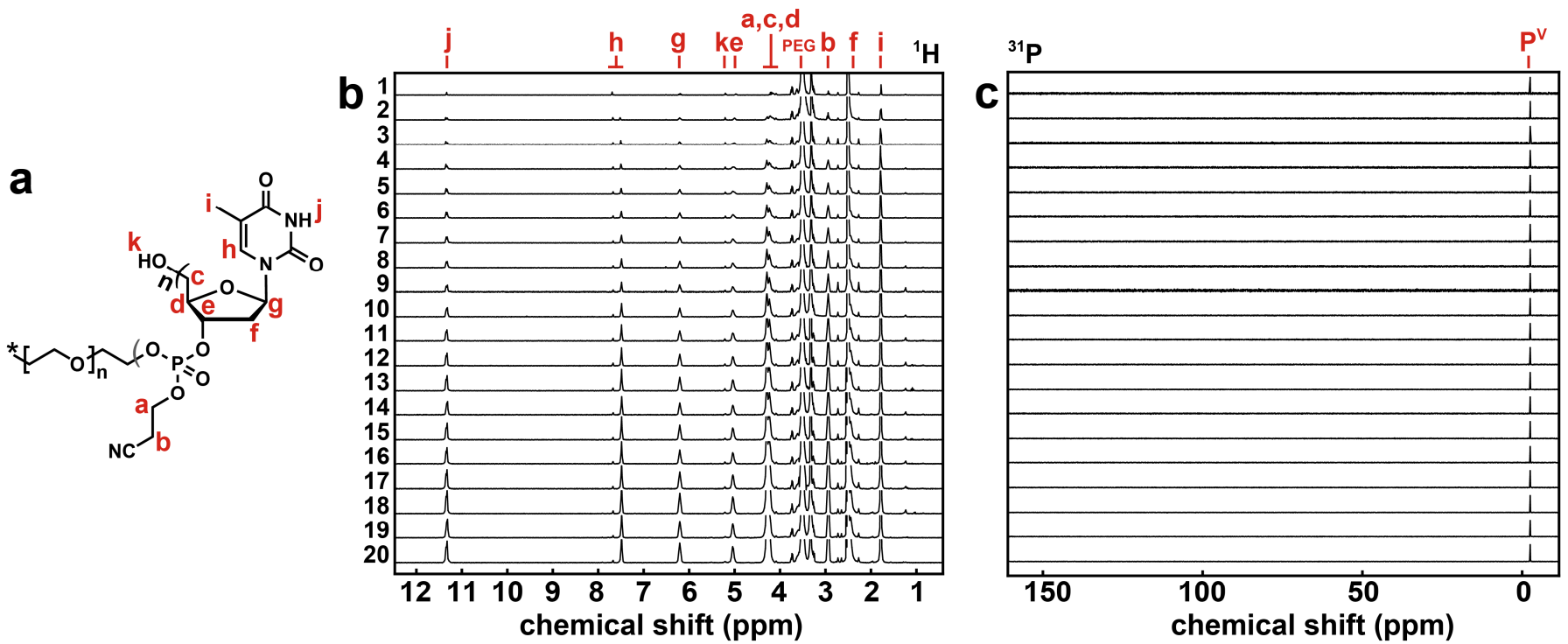

Figure S13. NMR-characterization of intermediate samples collected throughout the synthesis of 4-arm PEG$\mathrm{T}_{20}$ showing the growing oligonucleotide chain. (a) Chemical structure of 4-arm PEG- $\mathrm{T}_{\mathrm{n}}$ (DMT-off) with the cyanoethyl protecting group on the phosphate. (b) ${ }^{1} \mathrm{H}-\mathrm{NMR}$ (DMSO- $\mathrm{d}_{6}$ ) or 4-arm PEG-T $(D M T-$ off) with $\mathrm{n}=1$ to 20 normalized to the PEG peak, and (c) corresponding ${ }^{31} \mathrm{P}-\mathrm{NMR}$ normalized to the phosphate peak.

\section{NMR characterization of 4-arm PEG-T ${ }_{20}$ after precipitation}
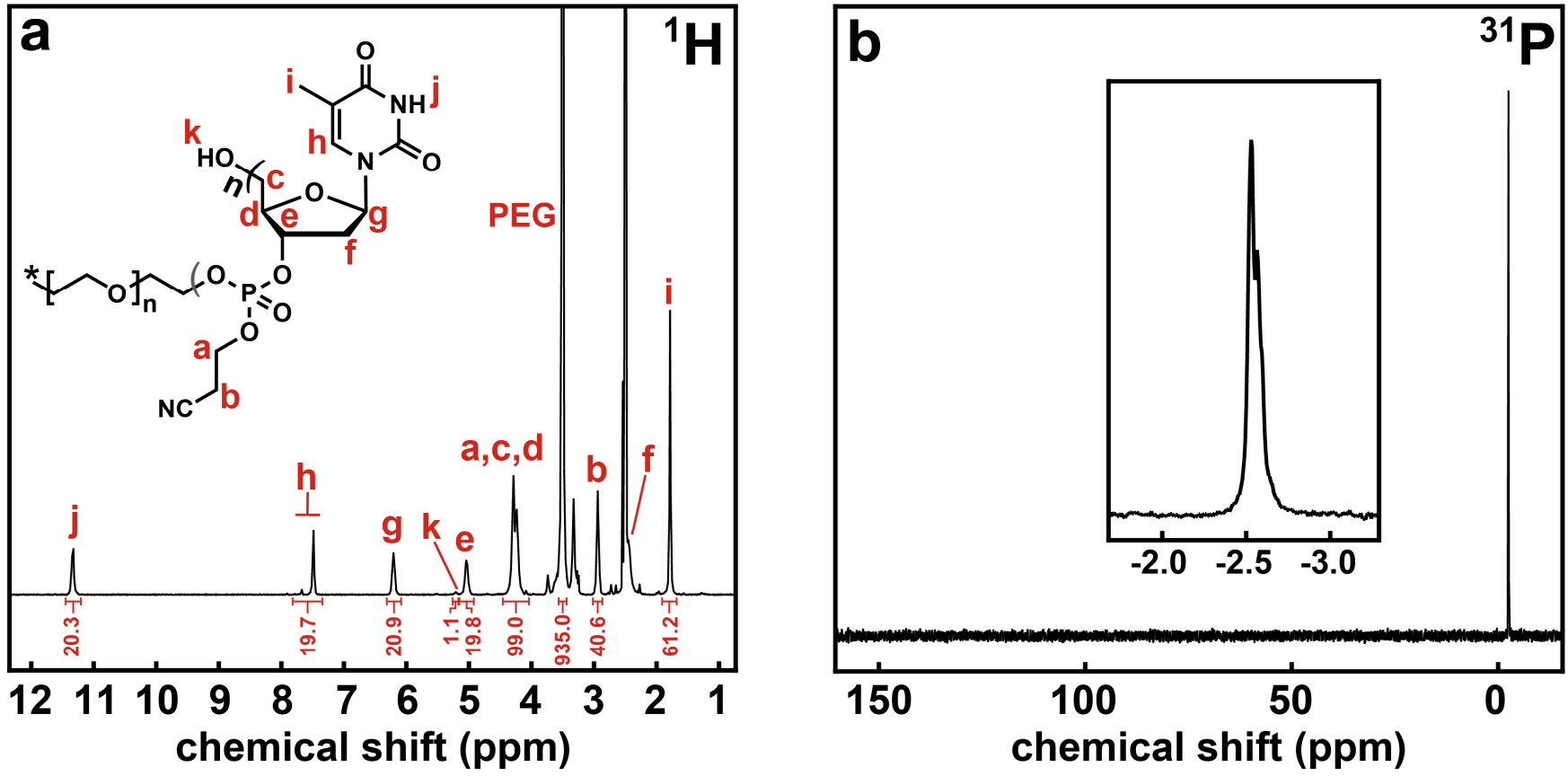

Figure S14. (a) ${ }^{1} \mathrm{H}-\mathrm{NMR}$ (DMSO- $\mathrm{d}_{6}$ ) of 4-arm PEG-T 20 with integrals (compared to $935 \mathrm{H}$ peak of a single arm of the 4-arm PEG-core) and (b) corresponding ${ }^{31} \mathrm{P}-\mathrm{NMR}$ with an inset showing the phosphate peak in detail. 
Table S1. Analysis of 4-arm PEG- $\mathrm{T}_{n}{ }^{1} \mathrm{H}-\mathrm{NMR}$ samples in DMSO- $\mathrm{d}_{6}$ measured after each nucleotide addition step.

\begin{tabular}{cccc}
\hline Step & $\begin{array}{c}\text { Thymine-3H } \\
\text { integral }^{\mathrm{a}}\end{array}$ & $\begin{array}{c}\text { apparent } \\
\text { number of } \mathrm{T}^{\mathrm{b}}\end{array}$ & $\begin{array}{c}\text { total apparent } \\
\text { coupling }(\%)^{\mathrm{c}}\end{array}$ \\
\hline 1 & 2.99 & 1.00 & 99.7 \\
2 & 5.98 & 1.99 & 99.7 \\
3 & 8.98 & 2.99 & 99.8 \\
4 & 12.15 & 4.05 & 101.3 \\
5 & 15.08 & 5.03 & 100.5 \\
6 & 18.24 & 6.08 & 101.3 \\
7 & 21.01 & 7.00 & 100.0 \\
8 & 24.10 & 8.03 & 100.4 \\
9 & 27.22 & 9.07 & 100.8 \\
10 & 30.36 & 10.12 & 101.2 \\
11 & 33.66 & 11.22 & 102.0 \\
12 & 36.69 & 12.23 & 101.9 \\
13 & 40.22 & 13.41 & 103.1 \\
14 & 43.09 & 14.36 & 102.6 \\
15 & 45.99 & 15.33 & 102.2 \\
16 & 49.22 & 16.41 & 102.5 \\
17 & 52.42 & 17.47 & 102.8 \\
18 & 55.46 & 18.49 & 102.7 \\
19 & 59.17 & 19.72 & 103.8 \\
20 & 61.24 & 20.41 & 102.1 \\
\hline
\end{tabular}

a Compared to the $935 \mathrm{H}$ of the PEG-core.

${ }^{c}$ The integral of thymine-3H divided by 3 .

c The number of $T$ relative to PEG divided by the reaction step number. 
Table S2. Mass of the product before and after each reaction step, including the amount of sample collected for analysis after each step.

\begin{tabular}{ccccccc}
\hline Step & $\begin{array}{c}M_{\mathrm{n}} \text { protected } \\
\text { PEG-T } \\
(\mathrm{g} / \mathrm{mol})^{\mathrm{a}}\end{array}$ & $\begin{array}{c}\text { weight before } \\
\text { reaction }(\mathrm{g})\end{array}$ & $\begin{array}{c}\text { weight after } \\
\text { reaction }(\mathrm{g})\end{array}$ & $\begin{array}{c}\text { nPEG-DNA } \\
(\mu \mathrm{mol})\end{array}$ & $\begin{array}{c}\text { recovered yield } \\
(\%)\end{array}$ & $\begin{array}{c}\text { sample } \\
\text { collected }(\mathrm{mg})\end{array}$ \\
\hline 0 & 41,170 & & & 459 & & \\
1 & 42,599 & 18.9 & 19.3 & 454 & 98.8 & 61 \\
2 & 44,024 & 19.3 & 19.1 & 435 & 96.0 & 50 \\
3 & 45,450 & 19.1 & 19.2 & 424 & 97.7 & 53 \\
4 & 46,875 & 19.2 & 19.7 & 419 & 99.3 & 49 \\
5 & 48,300 & 19.6 & 19.5 & 404 & 96.5 & 50 \\
6 & 49,725 & 19.5 & 19.6 & 393 & 97.7 & 47 \\
7 & 51,150 & 19.5 & 19.6 & 384 & 97.7 & 78 \\
8 & 52,576 & 19.5 & 19.7 & 376 & 98.3 & 63 \\
9 & 54,001 & 19.7 & 19.6 & 364 & 97.2 & 50 \\
10 & 55,426 & 19.6 & 18.9 & 341 & 94.1 & 4702 \\
11 & 56,851 & 14.2 & 14.0 & 247 & 96.1 & 46 \\
12 & 58,276 & 14.0 & 14.1 & 242 & 98.5 & 40 \\
13 & 59,702 & 14.1 & 14.0 & 234 & 97.0 & 37 \\
14 & 61,127 & 13.9 & 14.2 & 232 & 99.2 & 51 \\
15 & 62,552 & 14.1 & 14.1 & 226 & 97.9 & 3976 \\
16 & 63,977 & 10.2 & 10.2 & 160 & 98.5 & 231 \\
17 & 65,402 & 10.0 & 9.6 & 147 & 94.3 & 219 \\
18 & 66,828 & 9.4 & 9.3 & 140 & 96.8 & 283 \\
19 & 68,253 & 9.0 & 9.0 & 132 & 97.7 & 120 \\
20 & 69,678 & 8.9 & 9.0 & 129 & 99.1 & \\
\hline
\end{tabular}

a Calculated number-average molecular weight of 4-arm PEG-Tn (DMT-off, with cyanoethyl protecting group on the phosphate) 
Table S3. Analysis of analytical HPLC traces of deprotected 4-arm PEG- $T_{n}$ to quantify the amount of oligo-T impurities compounding during the synthesis. DMT-off phosphate-protected samples are deprotected in ammonia/methylamine (AMA) solution at $20 \mathrm{mg} / \mathrm{mL}$ for 30 minutes, followed by 200 -fold dilution with phosphate buffer.

\begin{tabular}{|c|c|c|c|c|c|}
\hline Step & $\begin{array}{c}M_{\mathrm{n}} \\
\text { deprotected } \\
\text { PEG-Tn } \\
(\mathrm{g} / \mathrm{mol})^{a}\end{array}$ & $\begin{array}{l}\text { DNA mass fraction } \\
\text { in PEG- } T_{n} \\
(\%)\end{array}$ & $\begin{array}{c}\text { PEG-Tn } \\
\text { peak integral } \\
(\%)^{b}\end{array}$ & $\begin{array}{c}\text { oligo-T } \\
\text { impurity integral } \\
(\%)^{c}\end{array}$ & $\begin{array}{c}\text { PEG-Tn } \\
\text { mass fraction } \\
(\%)^{d}\end{array}$ \\
\hline 1 & 42,387 & 2.9 & 94.6 & 5.4 & 99.8 \\
\hline 2 & 43,600 & 5.6 & 97.8 & 2.2 & 99.9 \\
\hline 3 & 44,813 & 8.1 & 98.7 & 1.3 & 99.9 \\
\hline 4 & 46,025 & 10.5 & 98.9 & 1.1 & 99.9 \\
\hline 5 & 47,238 & 12.8 & 98.8 & 1.2 & 99.9 \\
\hline 6 & 48,451 & 15.0 & 98.3 & 1.7 & 99.7 \\
\hline 7 & 49,664 & 17.1 & 97.7 & 2.3 & 99.6 \\
\hline 8 & 50,877 & 19.1 & 96.8 & 3.2 & 99.4 \\
\hline 9 & 52,090 & 21.0 & 95.9 & 4.1 & 99.1 \\
\hline 10 & 53,302 & 22.8 & 95.9 & 4.1 & 99.1 \\
\hline 11 & 54,515 & 24.5 & 95.3 & 4.7 & 98.8 \\
\hline 12 & 55,728 & 26.1 & 95.0 & 5.0 & 98.7 \\
\hline 13 & 56,941 & 27.7 & 94.7 & 5.3 & 98.5 \\
\hline 14 & 58,154 & 29.2 & 94.2 & 5.8 & 98.3 \\
\hline 15 & 59,367 & 30.7 & 94.1 & 5.9 & 98.2 \\
\hline 16 & 60,579 & 32.0 & 93.3 & 6.7 & 97.9 \\
\hline 17 & 61,792 & 33.4 & 93.1 & 6.9 & 97.7 \\
\hline 18 & 63,005 & 34.7 & 91.8 & 8.2 & 97.2 \\
\hline 19 & 64,218 & 35.9 & 88.9 & 11.1 & 96.0 \\
\hline 20 & 65,431 & 37.1 & 90.0 & 10.0 & 96.2 \\
\hline
\end{tabular}

a Calculated Number-average molecular weight of 4-arm PEG-Tn (DMT-off, phosphate deprotected).

${ }^{b}$ Integral of PEG-T in the HPLC trace divided by the integral of PEG- $T_{n}+$ oligo-T impurities.

${ }^{c}$ Integral of oligo-T impurities in the HPLC trace divided by the integral of PEG- $T_{n}+$ oligo-T impurities.

d Integral of PEG-Tn in the HPLC trace divided by the integral of PEG-Tn + oligo-T impurities, after the integral of PEG-Tn is divided by the DNA mass percentage of PEG-Tn in the respective step. This assumes the extinction coefficient of $T_{n}$ scales linearly with $n$, and that the product consists of 4-arm PEG- $T_{n}$ at step $n$. Note that a lower effective coupling would mean an increased purity owing to a lower DNA mass percentage in PEG-Tn. 
Table S4. Comparison of the T-to-PEG ratio obtained from ${ }^{1} \mathrm{H}-\mathrm{NMR}$ with the 4-arm PEG- $\mathrm{T}_{\mathrm{n}}$ purity obtained from HPLC analysis (by comparing the PEG- $T_{n}$ and oligo-T integral ratios, listed in Table S3) to determine the actual number of T nucleotides coupled to the 4-arm PEG.

\begin{tabular}{ccccc}
\hline Step & $\begin{array}{c}\text { NMR apparent } \\
\text { number of T per } \\
\text { PEG-arm }{ }^{\mathrm{a}}\end{array}$ & $\begin{array}{c}\text { HPLC molar } \\
\text { ratio PEG-Tn } \\
(\%)^{\mathrm{b}}\end{array}$ & $\begin{array}{c}\text { number of T per } \\
\text { PEG-arm } \\
(\%)^{\mathrm{c}}\end{array}$ & $\begin{array}{c}\text { total coupling } \\
(\%)^{\mathrm{d}}\end{array}$ \\
\hline 1 & 1.00 & 94.6 & 0.95 & 94.6 \\
2 & 1.99 & 97.8 & 1.96 & 97.8 \\
3 & 2.99 & 98.7 & 2.96 & 98.7 \\
4 & 4.05 & 98.9 & 3.96 & 98.9 \\
5 & 5.03 & 98.8 & 4.94 & 98.8 \\
6 & 6.08 & 98.3 & 5.90 & 98.3 \\
7 & 7.00 & 97.7 & 6.84 & 97.7 \\
8 & 8.03 & 96.8 & 7.74 & 96.8 \\
9 & 9.07 & 95.9 & 8.63 & 95.9 \\
10 & 10.12 & 95.9 & 9.68 & 96.8 \\
11 & 11.22 & 95.3 & 10.67 & 97.0 \\
12 & 12.23 & 95.0 & 11.68 & 97.4 \\
13 & 13.41 & 94.7 & 12.70 & 97.7 \\
14 & 14.36 & 94.2 & 13.66 & 97.6 \\
15 & 15.33 & 94.1 & 14.68 & 97.9 \\
16 & 16.41 & 93.3 & 15.58 & 97.4 \\
17 & 17.47 & 93.1 & 16.57 & 97.5 \\
18 & 18.49 & 91.8 & 17.35 & 96.4 \\
19 & 19.72 & 88.9 & 17.78 & 93.6 \\
20 & 20.41 & 90.0 & 18.94 & 94.7 \\
\hline
\end{tabular}

a The integral of thymine-3H compared to the 935H of the PEG-core divided by 3, see Table S2.

b Integral of PEG-Tn in the HPLC trace divided by the integral of PEG- $T_{n}+$ oligo-T impurities, see Table S3.

c Obtained by multiplying the apparent number of T-nucleotides per arm (NMR) by the PEG-Tn purity.

${ }^{d}$ Number of $T$ nucleotides per arm divided by the number of reaction steps. 
NMR characterization of HPLC-purified 4-arm PEG-T $10,15,20$

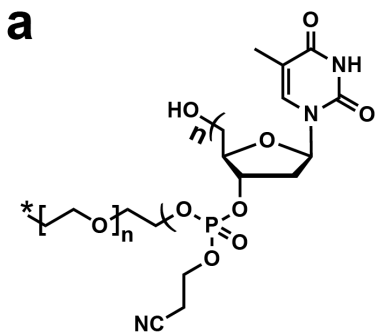

b

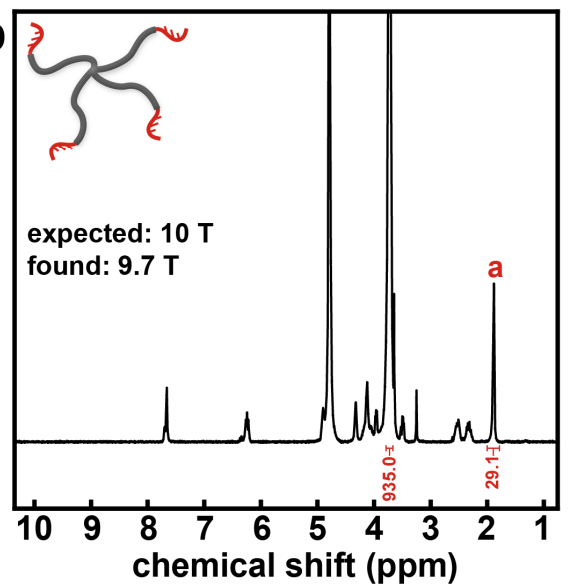

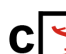
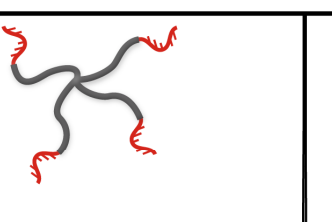

expected: $15 \mathrm{~T}$

found: $13.8 \mathrm{~T}$

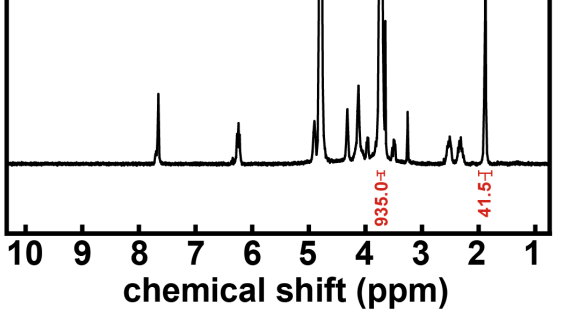

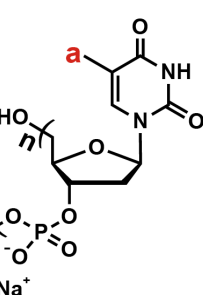

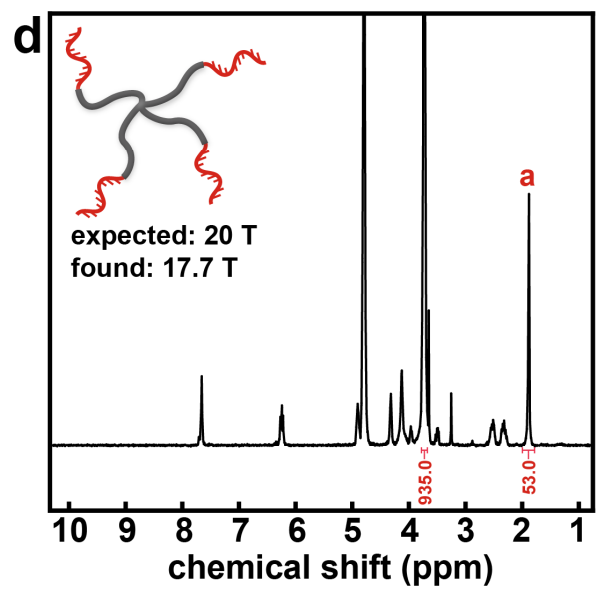

Figure S15. ${ }^{1} \mathrm{H}-\mathrm{NMR}$ characterization of 4 -arm PEG-T $10,-\mathrm{T}_{15}$ and $-\mathrm{T}_{20}$ after preparative HPLC purification. (a) Reaction scheme for the deprotection of the phosphate group (30 minutes in ammonia/methylamine (AMA) solution) followed by preparative HPLC purification and dialysis to exchange the triethylammonium counterions from the HPLC buffer to sodium ions. (b-d) ${ }^{1} \mathrm{H}-\mathrm{NMR}\left(\mathrm{D}_{2} \mathrm{O}\right)$ of 4-arm PEG- $\mathrm{T}_{10},-\mathrm{T}_{15}$ and $-\mathrm{T}_{20}$ after purification, showing the actual numbers of T nucleotides attached per PEG-arm as determined by comparing the thymine$3 \mathrm{H}$ integral to the $935 \mathrm{H}$ integral of a single PEG arm. 


\section{SEC analysis of 4-arm PEG-T 20}

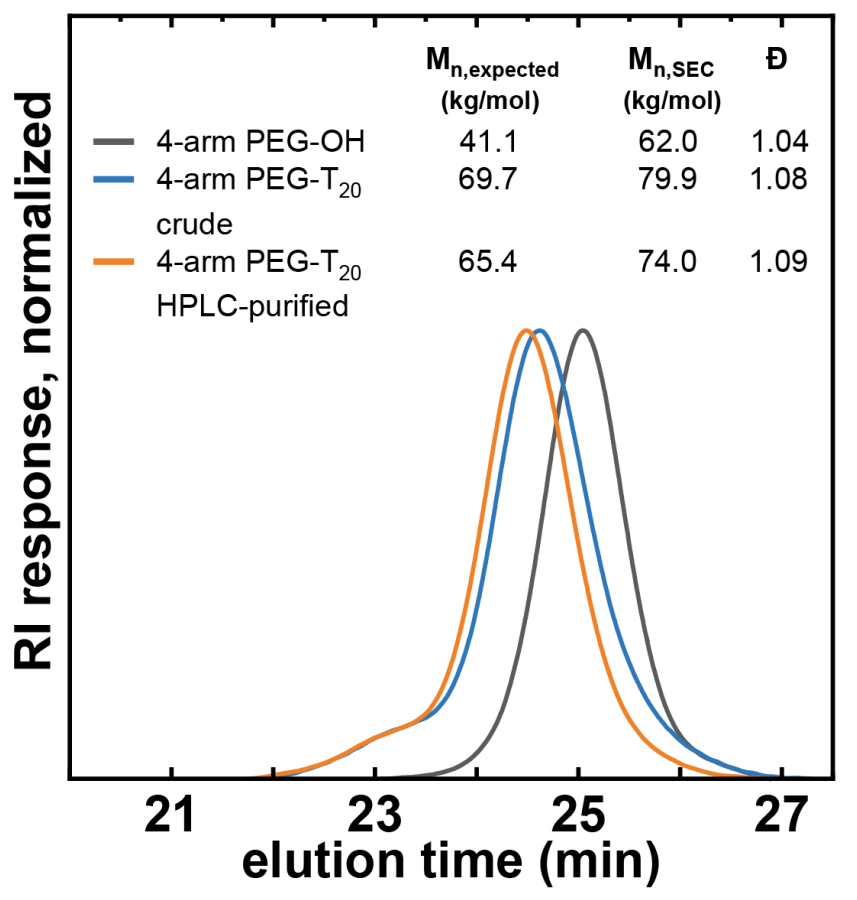

Figure S16. SEC (in DMAc $+0.5 \% \mathrm{LiBr}$ ) characterization of 4-arm PEG-OH, 4-arm PEG-T20 (phosphate protected), and 4-arm PEG-T 20 (deprotected, HPLC-purified).

\section{removal of phosphate-protecting group (CE) on 4-arm PEG-T}

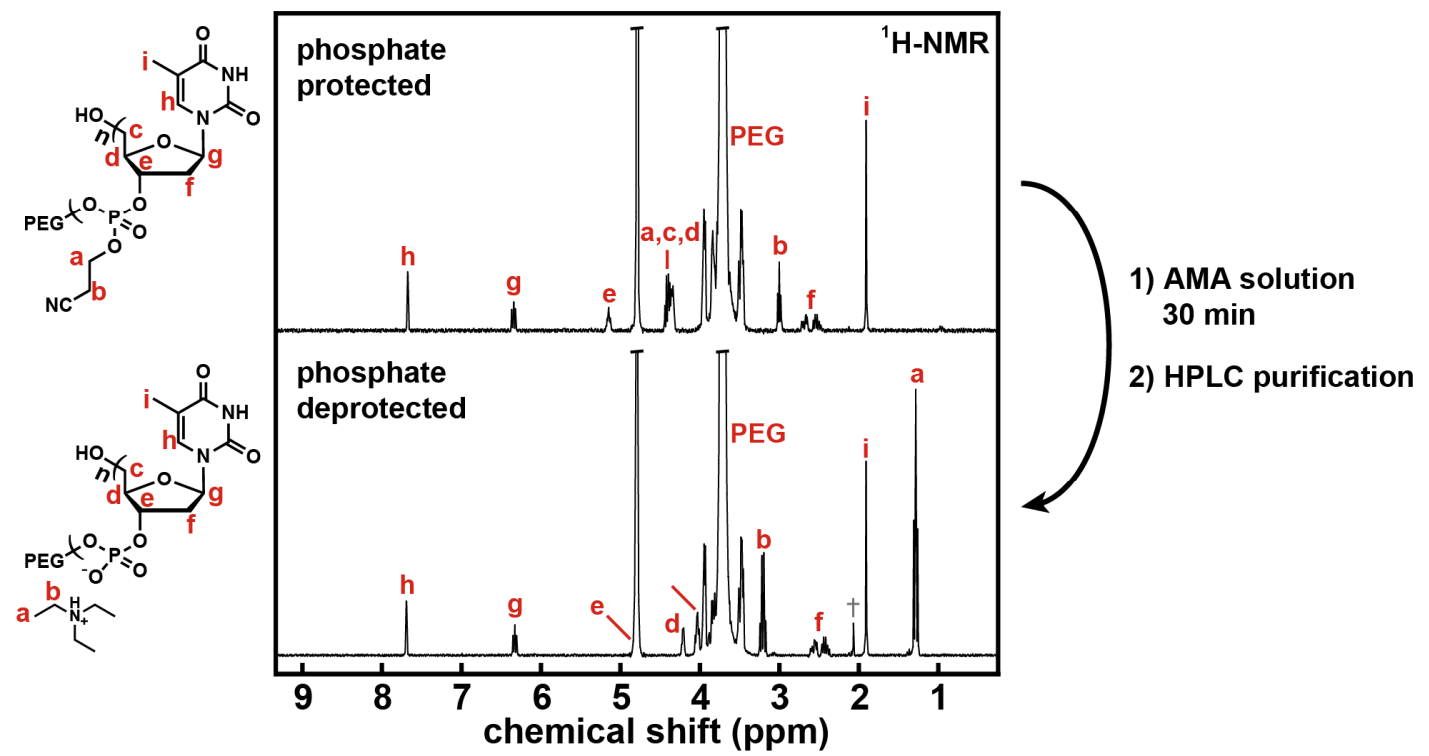

Figure S17. ${ }^{1} \mathrm{H}-\mathrm{NMR}\left(\mathrm{D}_{2} \mathrm{O}\right)$ of 4-arm PEG-dT-OH $\left(\mathrm{P}^{\mathrm{V}}\right)$ before (top) and after (bottom) deprotecting the phosphate by removing the CE-group in concentrated ammonia/methylamine (AMA) solution for 30 minutes at room temperature. The phosphate-deprotected sample was additionally purified by preparative HPLC, resulting in triethylammonium counter-ions. + Indicates residual acetate from the triethylamine-acetic acid buffer. 
comparison of 4-arm PEG-A obtained by OP-LPOS and literature procedure
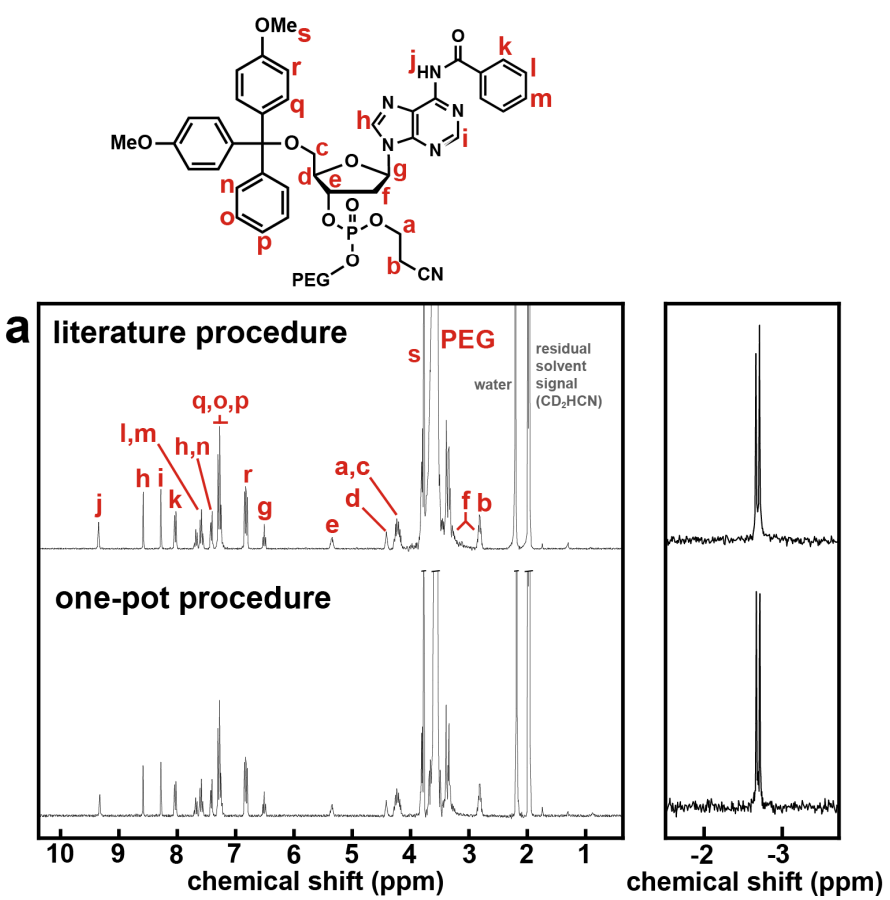
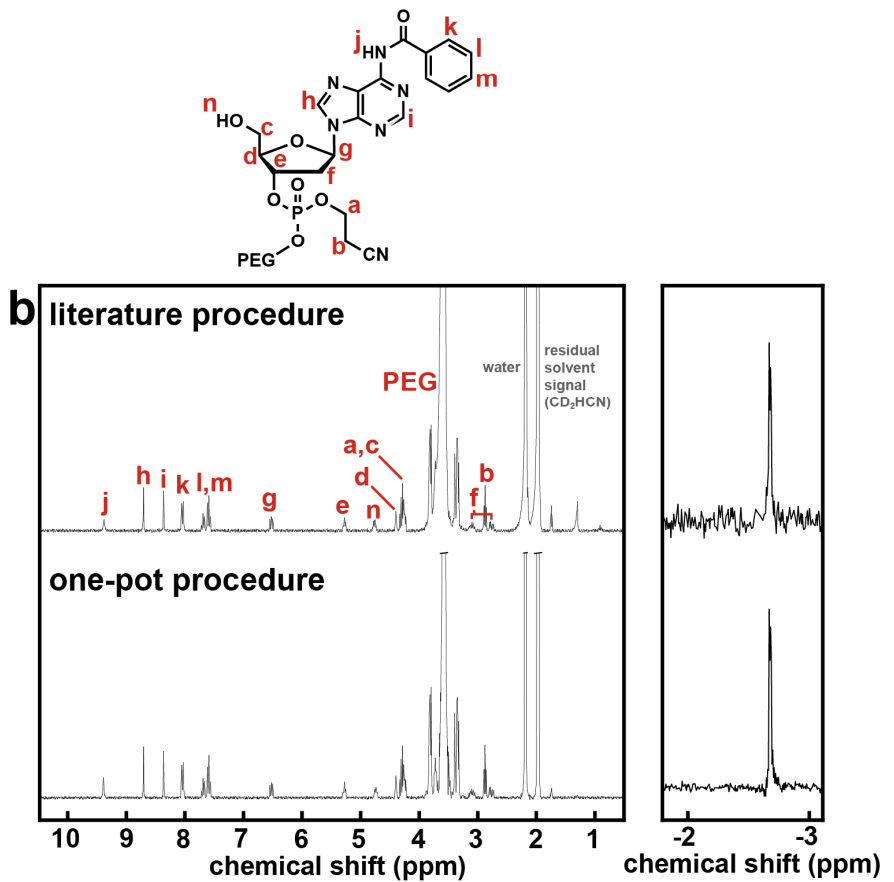

Figure S18. Comparison of ${ }^{1} \mathrm{H}-\mathrm{NMR}\left(\mathrm{CD}_{3} \mathrm{CN}\right)$ and corresponding ${ }^{31} \mathrm{P}-\mathrm{NMR}$ for (a) 4-arm PEG-dA(Bz)-DMT $\left(\mathrm{P}^{\mathrm{V}}\right)$ and (b) 4-arm PEG-dA(Bz)-OH $\left(\mathrm{P}^{\vee}\right)$, showing no difference between the respective products obtained by literature procedure (intermediate workup after coupling, oxidation, detritylation (2x)) or one-pot liquid-phase oligonucleotide synthesis. 
a<smiles>O=C(O)C(Cl)(Cl)Cl</smiles>

30 eq. to DMT room temperature

efficiency $=1092 \pm 57$<smiles>O=C(O)C(Cl)(Cl)Cl</smiles>

30 eq. to DMT $0^{\circ} \mathrm{C}$

efficiency $=2887 \pm 184$
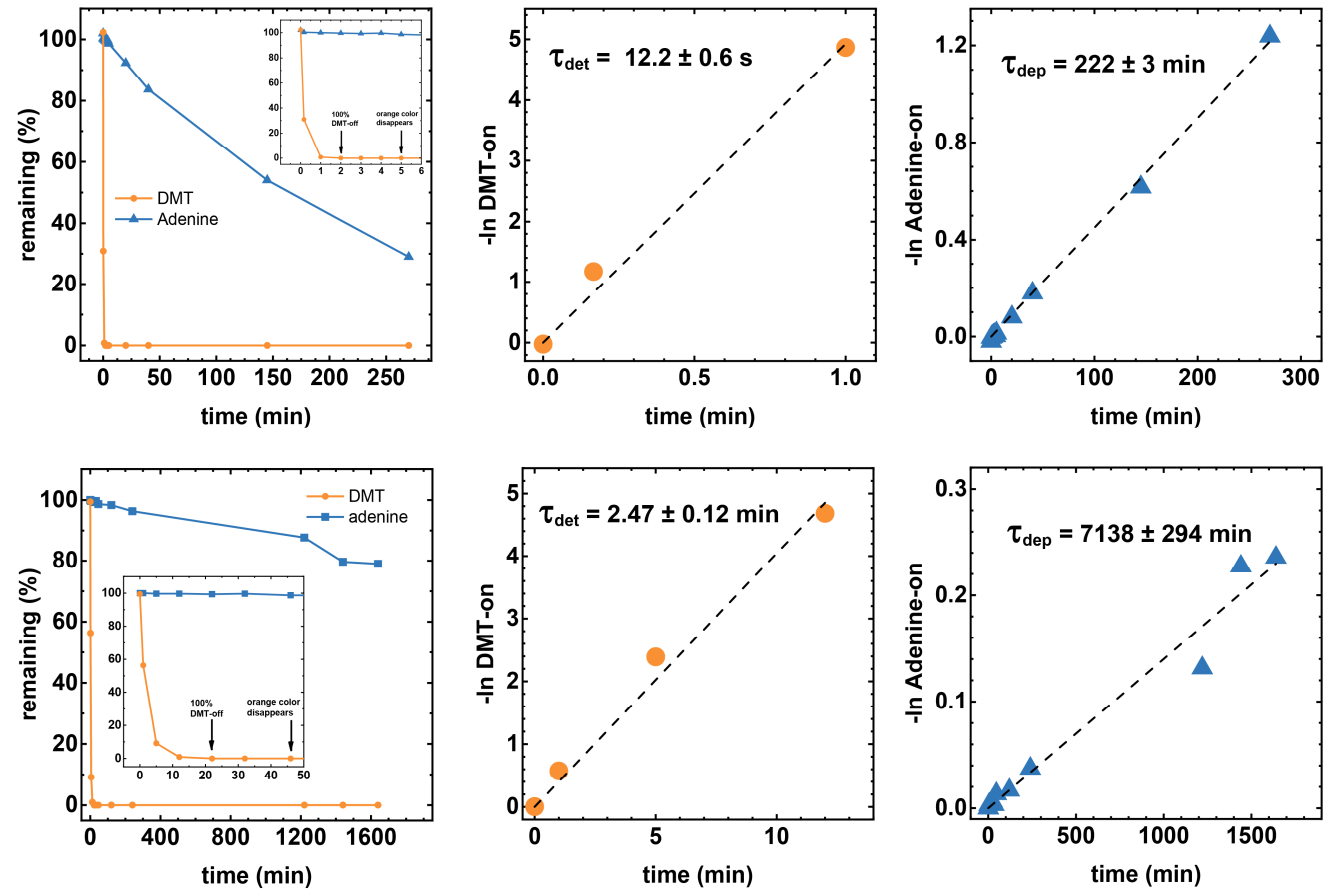

C<smiles>O=C(O)C(Cl)Cl</smiles>

60 eq. to DMT room temperature efficiency $=750 \pm 21$
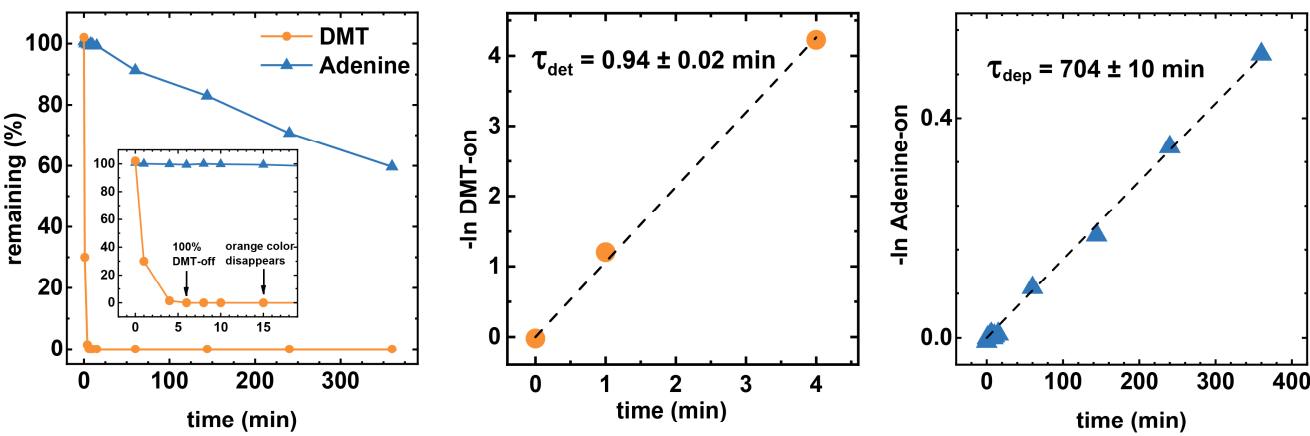<smiles>O=C(O)C(Cl)Cl</smiles>

60 eq. to DMT $0^{\circ} \mathrm{C}$

efficiency $=5519 \pm 503$
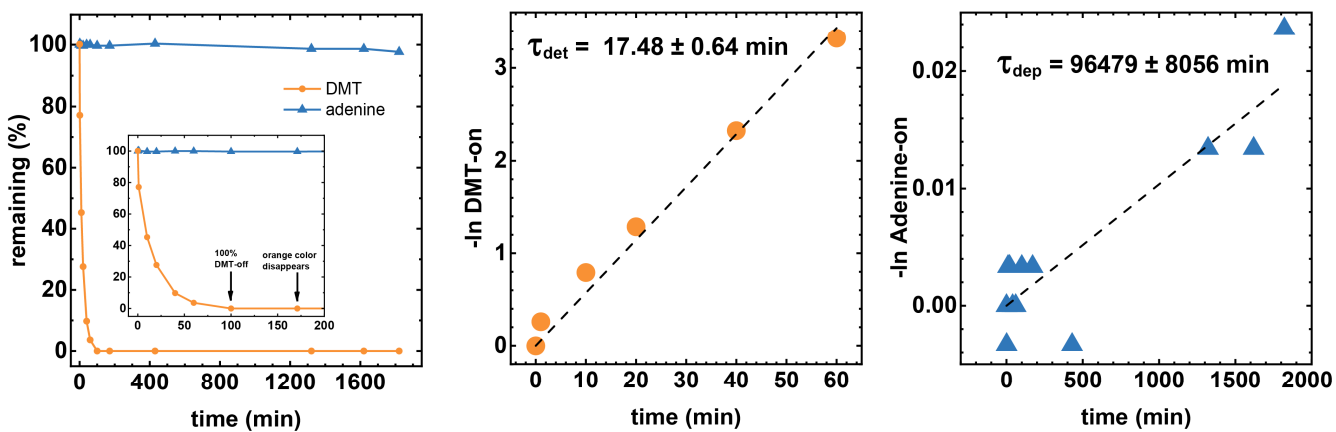

Figure S19. Kinetics and linearization for the detritylation and depurination reaction kinetics during a one-pot liquid phase addition of an A nucleotide with (a-b) 30 eq. TCA to DMT and (c-d) 60 eq. DCA to DMT at room temperature and $0^{\circ} \mathrm{C}$. A single experiment consists of a coupling step (3 eq. $\mathrm{A}(\mathrm{Bz})$ phosphoramidite and 12 eq. ETT to $\mathrm{OH}$ groups) for 60 minutes, oxidation at $0{ }^{\circ} \mathrm{C}$ for 10 minutes, followed by detritylation. Intermediate samples are collected by precipitating twice into $-30{ }^{\circ} \mathrm{C} 2$-propanol and washing with room temperature 2propanol. 
a<smiles>O=C(O)C(Cl)Cl</smiles>

50 eq. to DMT

$0^{\circ} \mathrm{C}$

efficiency $=2993 \pm 480$

b<smiles>O=C(O)C(Cl)Cl</smiles>

70 eq. to DMT

$0^{\circ} \mathrm{C}$

efficiency $=6115 \pm 555$
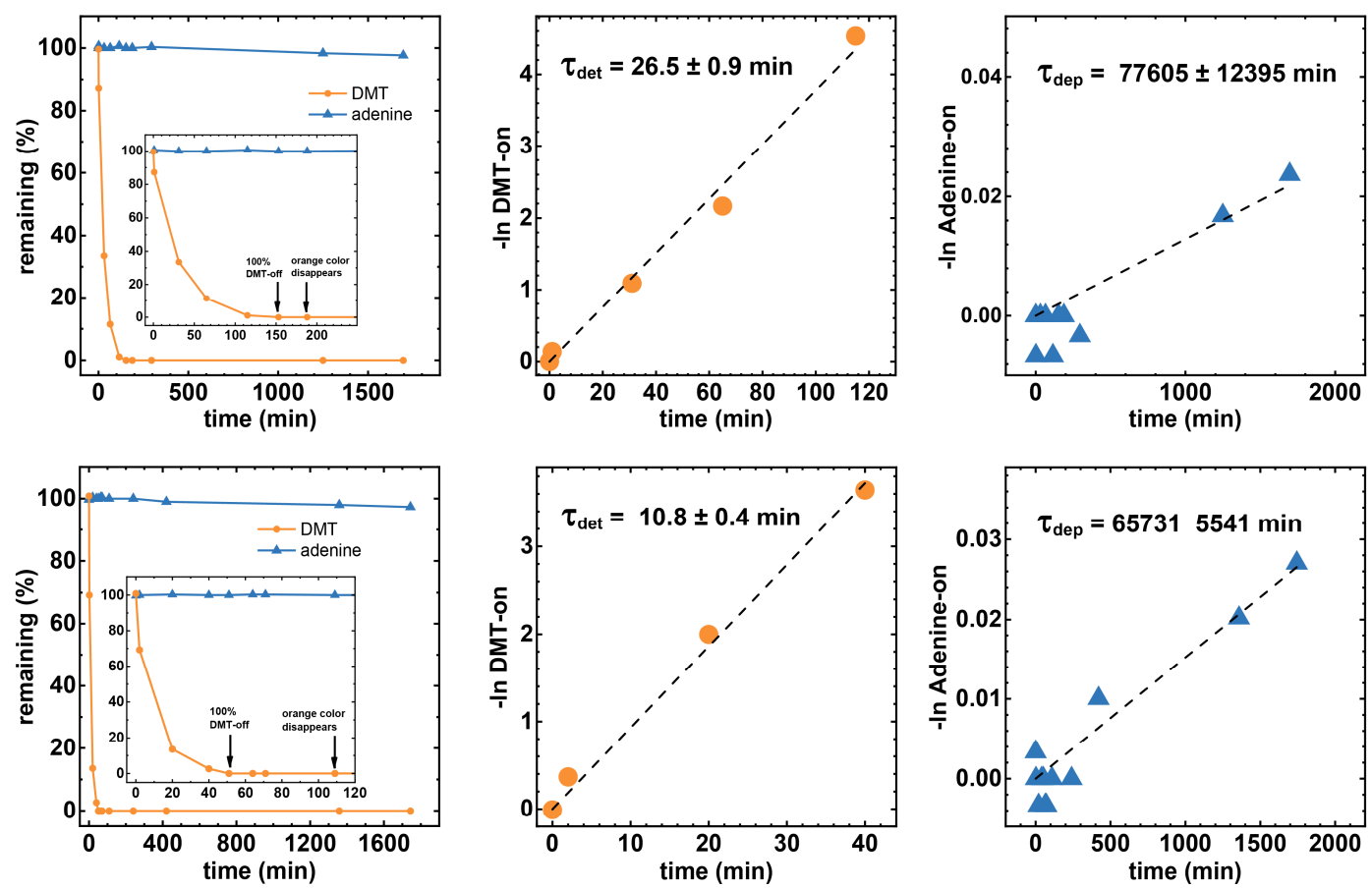

Figure S20. Kinetics and linearization for the detritylation and depurination reaction kinetics during a one-pot liquid phase addition of an A nucleotide with (a) 50 and (b) 70 eq. DCA to DMT at $0{ }^{\circ} \mathrm{C}$. A single experiment consists of a coupling step ( 3 eq. $A(B z)$ phosphoramidite and 12 eq. ETT to OH groups) for 60 minutes, oxidation at $0{ }^{\circ} \mathrm{C}$ for 10 minutes, followed by detritylation. Intermediate samples are collected by precipitating twice into $30{ }^{\circ} \mathrm{C} 2$-propanol and washing with room temperature 2-propanol.

\section{Conditions}

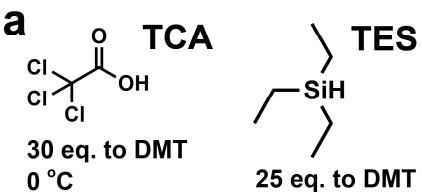

efficiency $=2887 \pm 184$

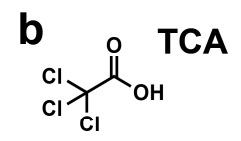

30 eq. to DMT

$0{ }^{\circ} \mathrm{C}$

efficiency $=2364 \pm 477$
Reaction Kinetics
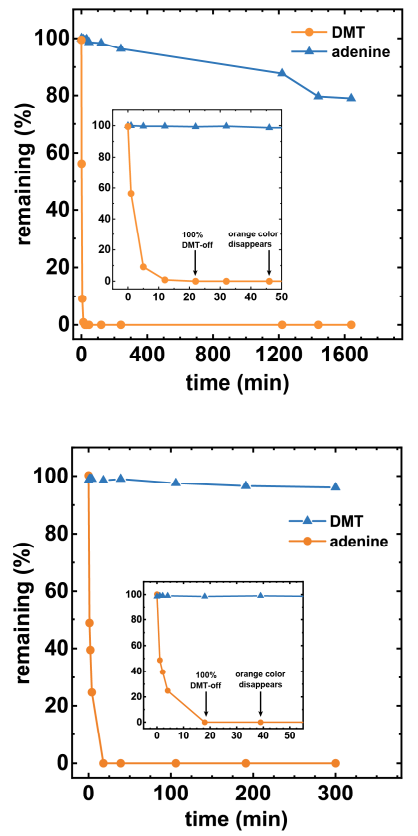

Detritylation
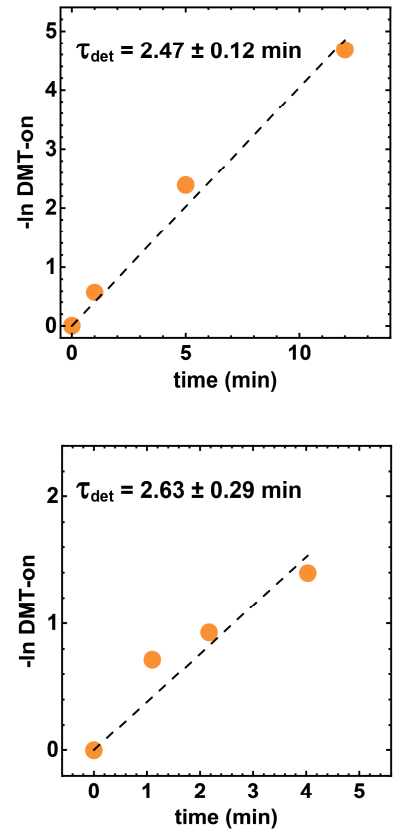

Depurination
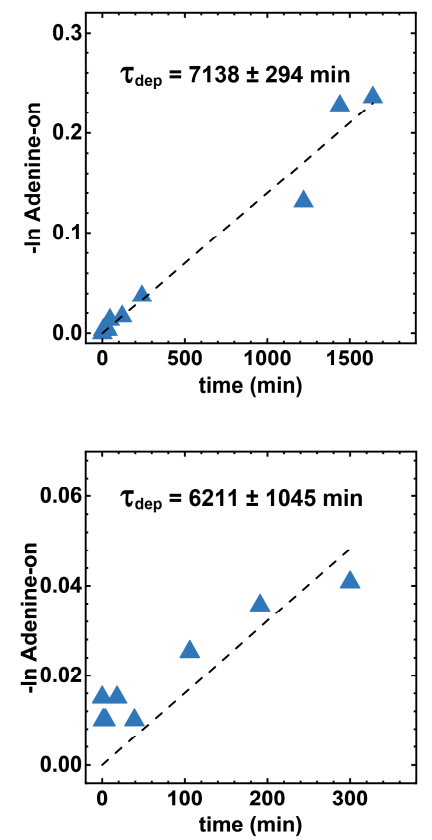
Figure S21. Kinetics and linearization for the detritylation and depurination reaction kinetics during a one-pot liquid phase addition of an A nucleotide with (a) triethyl silane (TES) and (b) dimethyl ethyl silane (DMES). Conditions: Coupling with 3 eq. $A(B z)$ phosphoramidite and 12 eq. ETT to $\mathrm{OH}$ groups for 60 minutes. Oxidation at $0^{\circ} \mathrm{C}$ for 10 minutes. Detritylation with 30 eq. TCA and 25 eq. silane (TES or DMES) to DMT at $0{ }^{\circ} \mathrm{C}$. Samples are collected by precipitating twice into $-30^{\circ} \mathrm{C} 2$-propanol and washing with room temperature 2-propanol, then diethyl ether.

\section{Calculation of errors for detritylation efficiency}

For single time constants $\tau_{\text {dep }}$ and $\tau_{\text {det, }}$ the standard deviation is obtained from the linear fit of the linearized first order kinetic plot for the percentage of adenine and DMT respectively. The error on the efficiency defined as $E$ $=\tau_{\text {dep }} / \tau_{\text {det }}$ is determined as the independent propagation of errors (equation 1 ).

$$
\sigma_{E}=\sqrt{\left(\frac{\partial E}{\partial \tau_{d e p}} \sigma_{\tau_{d e p}}\right)^{2}+\left(\frac{\partial E}{\partial \tau_{d e t}} \sigma_{\tau_{d e t}}\right)^{2}}=\sqrt{\left(\frac{1}{\tau_{d e t}} \sigma_{\tau_{d e p}}\right)^{2}+\left(-\frac{\tau_{d e p}}{\tau_{d e t}{ }^{2}} \sigma_{\tau_{d e t}}\right)^{2}}
$$


Table S5. Mass of the 4-arm PEG- $A_{n}$ product before and after each reaction step, including the amount of sample collected for analysis after each step.

\begin{tabular}{|c|c|c|c|c|c|c|}
\hline Step & $\begin{array}{c}M_{n} \text { protected } \\
\text { PEG-A } \\
(\mathrm{g} / \mathrm{mol})^{\mathrm{a}}\end{array}$ & $\begin{array}{l}\text { weight before } \\
\text { reaction }(\mathrm{g})\end{array}$ & $\begin{array}{l}\text { weight after } \\
\text { reaction }(g)\end{array}$ & $\begin{array}{l}\mathrm{n}_{\text {PEG-DNA }} \\
(\mu \mathrm{mol})\end{array}$ & $\begin{array}{l}\text { recovered step } \\
\text { yield (\%) }\end{array}$ & $\begin{array}{c}\text { sample } \\
\text { collected (mg) }\end{array}$ \\
\hline 0 & 41,170 & & & 245 & & \\
\hline 1 & 43,055 & 10.1 & 10.0 & 232 & 94.9 & 90 \\
\hline 2 & 44,937 & 9.9 & 10.1 & 224 & 97.4 & 91 \\
\hline 3 & 46,818 & 10.0 & 10.1 & 216 & 97.4 & 100 \\
\hline 4 & 48,700 & 10.0 & 10.2 & 209 & 97.7 & 138 \\
\hline 5 & 50,581 & 10.0 & 10.1 & 200 & 97.2 & 593 \\
\hline 6 & 52,463 & 9.5 & 9.5 & 182 & 96.4 & 107 \\
\hline 7 & 54,344 & 9.4 & 9.5 & 176 & 97.6 & 139 \\
\hline 8 & 56,226 & 9.4 & 9.5 & 170 & 98.1 & 119 \\
\hline 9 & 58,107 & 9.4 & 9.5 & 163 & 97.3 & 124 \\
\hline 10 & 59,989 & 9.3 & 9.4 & 157 & 97.7 & 99 \\
\hline 11 & 61,870 & 9.3 & 9.3 & 150 & 96.3 & 131 \\
\hline 12 & 63,752 & 9.1 & 9.0 & 142 & 95.9 & 132 \\
\hline 13 & 65,633 & 8.9 & 8.8 & 134 & 95.8 & 108 \\
\hline 14 & 67,515 & 8.7 & 8.6 & 127 & 95.8 & 738 \\
\hline 15 & 69,396 & 7.8 & 7.0 & 101 & 86.9 & 1106 \\
\hline 16 & 71,278 & 5.9 & 5.5 & 75 & 88.9 & 62 \\
\hline 17 & 73,159 & 5.3 & 4.9 & 66 & 89.3 & 75 \\
\hline 18 & 75,041 & 4.8 & 4.5 & 60 & 92.4 & 71 \\
\hline 19 & 76,922 & 4.5 & 4.0 & 52 & 88.0 & 115 \\
\hline 20 & 78,804 & 3.9 & 3.4 & 43 & 84.7 & \\
\hline
\end{tabular}

a Calculated number-average molecular weight of 4-arm PEG-An (DMT-off, with Bz- and CE-protecting groups). 


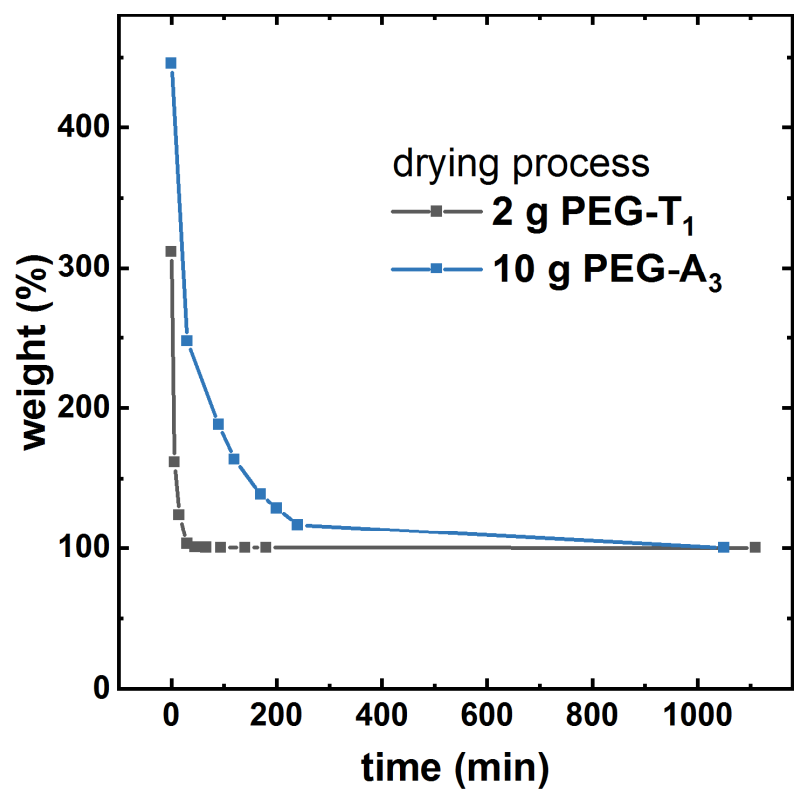

Figure S22. Comparison of the drying process under high vacuum for a batch of $2 \mathrm{~g} \mathrm{PEG-T_{1 }}$ and a $10 \mathrm{~g}$ batch of PEG-A $A_{3}$. Both were precipitated into cold 2-propanol, washed with 2-propanol, and washed with excess room temperature diethyl ether. Weight is listed as relative to the product dried overnight under vacuum.

\section{NMR characterization of intermediate samples during 4-arm PEG-A ${ }_{20}$ synthesis}
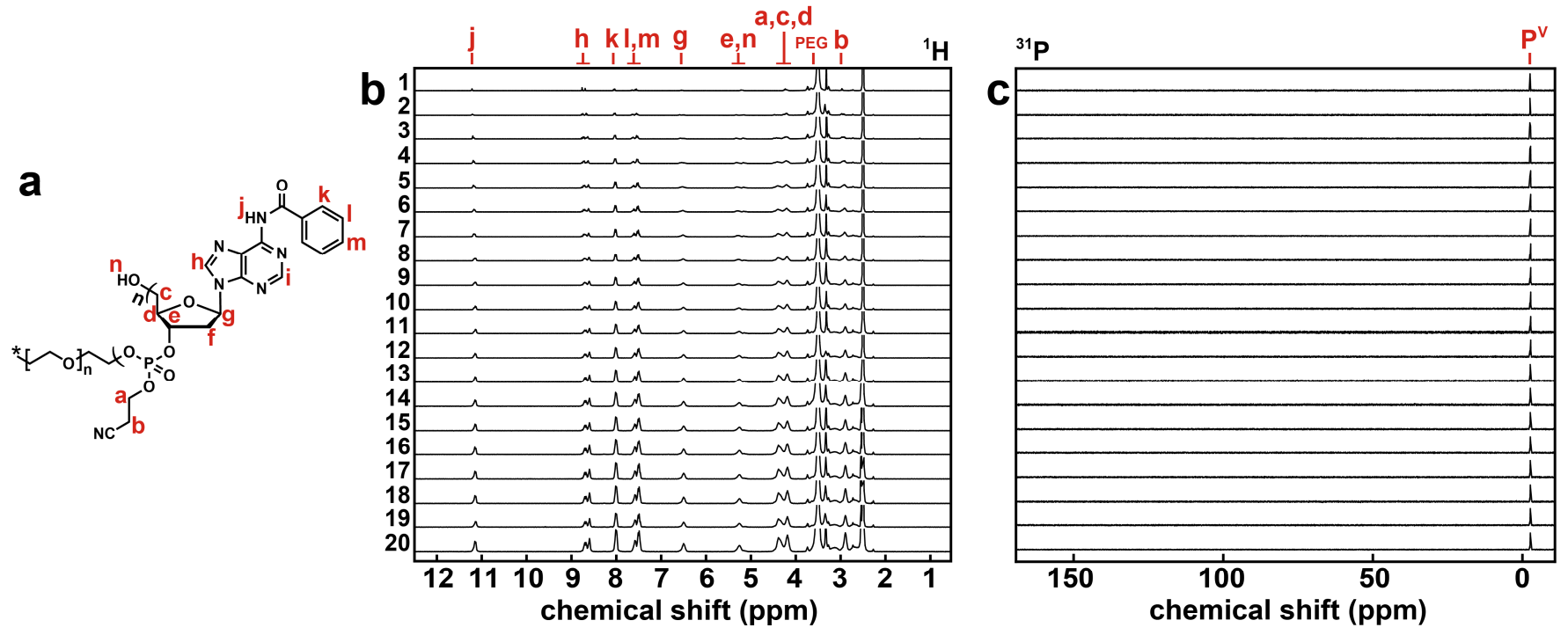

Figure S23. NMR-characterization of intermediate samples collected throughout the synthesis of 4-arm PEG$A_{20}$ showing the growing oligonucleotide chain. (a) Chemical structure of 4-arm PEG- $A_{n}$ (DMT-off) with the Bzand CE-phosphate protecting group. (b) ${ }^{1} \mathrm{H}-\mathrm{NMR}$ (DMSO- $\mathrm{d}_{6}$ ) or 4-arm PEG-An (DMT-off) with $\mathrm{n}=1$ to 20 normalized to the PEG peak, and (c) corresponding ${ }^{31} \mathrm{P}-\mathrm{NMR}$ normalized to the phosphate peak. 
NMR characterization of 4-arm PEG-A $A_{20}$ after precipitation
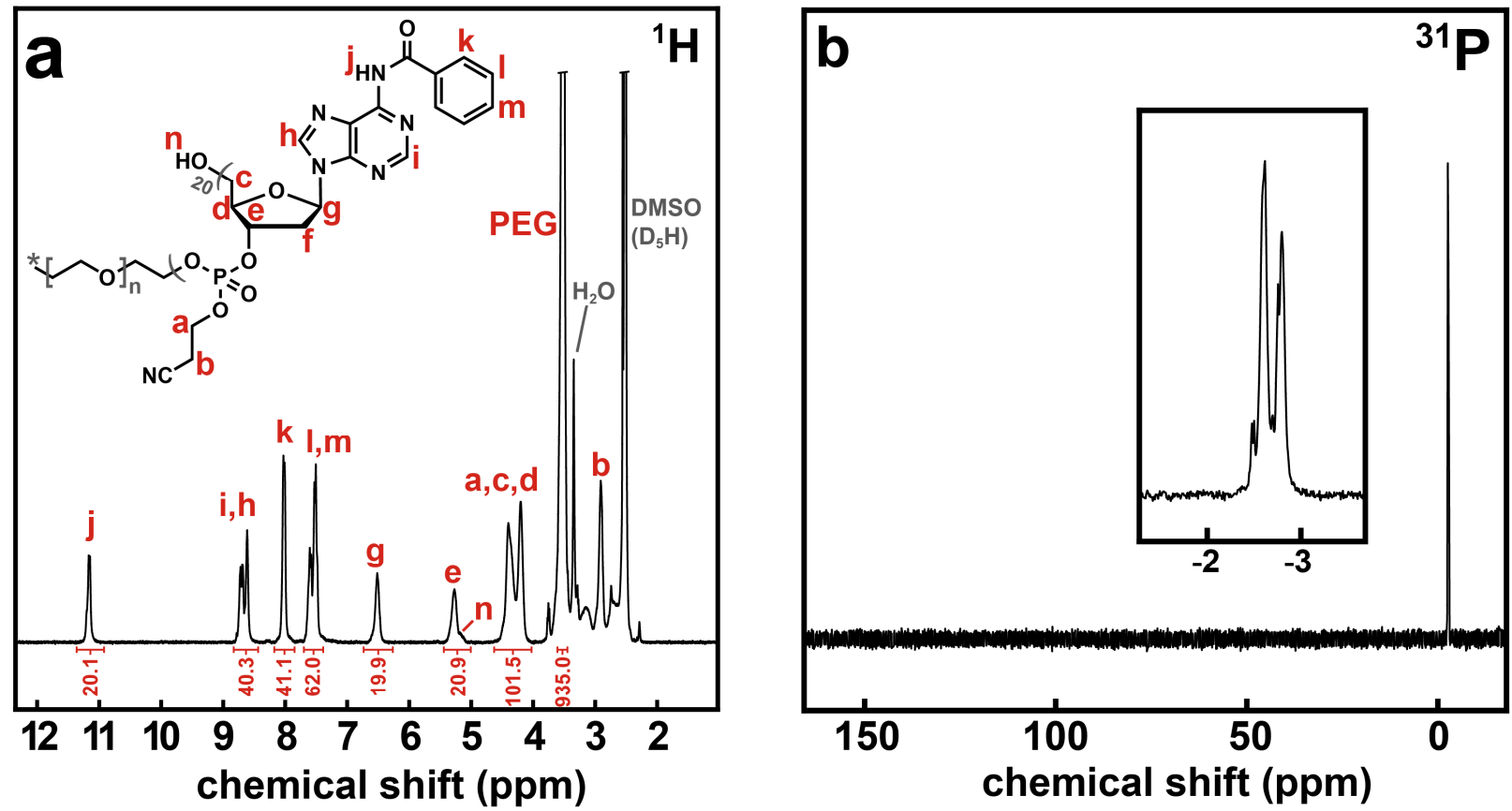

Figure S24. (a) ${ }^{1} \mathrm{H}-\mathrm{NMR}\left(\mathrm{DMSO}-\mathrm{d}_{6}\right.$ ) of crude 4-arm PEG-A 20 with integrals (compared to $935 \mathrm{H}$ peak of a single arm of the 4-arm PEG-core) and (b) corresponding ${ }^{31} \mathrm{P}-\mathrm{NMR}$ with an inset showing the phosphate peak in detail. 
Table 6. Analysis of 4-arm PEG-A ${ }_{n}{ }^{1} \mathrm{H}-\mathrm{NMR}$ samples in DMSO- $\mathrm{d}_{6}$ measured after each nucleotide addition step.

\begin{tabular}{|c|c|c|c|}
\hline Step & $\begin{array}{c}\text { Adenine-Bz-2H } \\
\text { integral }^{\mathrm{a}}\end{array}$ & $\begin{array}{c}\text { apparent } \\
\text { number of } A{ }^{b}\end{array}$ & $\begin{array}{l}\text { total apparent } \\
\text { coupling }(\%)^{\circ}\end{array}$ \\
\hline 1 & 1.95 & 0.98 & 97.5 \\
\hline 2 & 3.96 & 1.98 & 99.0 \\
\hline 3 & 5.96 & 2.98 & 99.3 \\
\hline 4 & 7.93 & 3.97 & 99.1 \\
\hline 5 & 9.82 & 4.91 & 98.2 \\
\hline 6 & 11.88 & 5.94 & 99.0 \\
\hline 7 & 14.09 & 7.05 & 100.6 \\
\hline 8 & 15.98 & 7.99 & 99.9 \\
\hline 9 & 18.09 & 9.05 & 100.5 \\
\hline 10 & 20.18 & 10.09 & 100.9 \\
\hline 11 & 22.20 & 11.10 & 100.9 \\
\hline 12 & 24.38 & 12.19 & 101.6 \\
\hline 13 & 26.45 & 13.23 & 101.7 \\
\hline 14 & 28.78 & 14.39 & 102.8 \\
\hline 15 & 30.79 & 15.40 & 102.6 \\
\hline 16 & 32.97 & 16.49 & 103.0 \\
\hline 17 & 34.95 & 17.48 & 102.8 \\
\hline 18 & 37.06 & 18.53 & 102.9 \\
\hline 19 & 39.22 & 19.61 & 103.2 \\
\hline 20 & 41.09 & 20.55 & 102.7 \\
\hline
\end{tabular}

a Compared to the $935 \mathrm{H}$ of the PEG-core.

c The integral of the adenine benzoyl-2H divided by 2 .

c The number of $A$ relative to $P E G$ divided by the reaction step number. 
Table S7. Analysis of analytical HPLC traces of deprotected 4-arm PEG- $A_{n}$ to quantify the amount of oligo-A impurities compounding during the synthesis. The (Bz- and CE-protected) samples were deprotected in ammonia/methylamine (AMA) solution at $20 \mathrm{mg} / \mathrm{mL}$ for 120 minutes, followed by 200 -fold dilution with phosphate buffer.

\begin{tabular}{cccccc}
\hline Step & $\begin{array}{c}M_{n} \\
\text { deprotected } \\
\text { PEG- } A_{n} \\
(\mathrm{~g} / \mathrm{mol})^{\mathrm{a}}\end{array}$ & $\begin{array}{c}\text { DNA mass fraction } \\
\text { in PEG- } A_{n} \\
(\%)\end{array}$ & $\begin{array}{c}\text { PEG-A } A_{n} \\
\text { peak integral } \\
(\%)^{\mathrm{b}}\end{array}$ & $\begin{array}{c}\text { oligo-A } \\
\text { impurity integral } \\
(\%)^{\mathrm{c}}\end{array}$ & $\begin{array}{c}\text { PEG-A } \\
\text { mass fraction } \\
(\%)^{\mathrm{d}}\end{array}$ \\
\hline 1 & 42,427 & 3.0 & 97.7 & 2.3 & 99.9 \\
2 & 43,680 & 5.7 & 98.6 & 1.4 & 99.9 \\
3 & 44,933 & 8.4 & 99.0 & 1.0 & 99.9 \\
4 & 46,185 & 10.9 & 98.5 & 1.5 & 99.8 \\
5 & 47,438 & 13.2 & 98.3 & 1.7 & 99.8 \\
6 & 48,691 & 15.4 & 98.1 & 1.9 & 99.7 \\
7 & 49,944 & 17.6 & 97.5 & 2.5 & 99.5 \\
8 & 51,197 & 19.6 & 96.8 & 3.2 & 99.4 \\
9 & 52,450 & 21.5 & 95.6 & 4.4 & 99.0 \\
10 & 53,702 & 23.3 & 94.2 & 5.8 & 98.6 \\
11 & 54,955 & 25.1 & 91.9 & 8.1 & 97.8 \\
12 & 56,208 & 26.8 & 90.6 & 9.4 & 97.3 \\
13 & 57,461 & 28.4 & 89.8 & 10.2 & 96.9 \\
14 & 58,714 & 29.9 & 88.5 & 11.5 & 96.3 \\
15 & 59,967 & 31.3 & 87.4 & 12.6 & 95.7 \\
16 & 61,219 & 32.8 & 87.6 & 12.4 & 95.6 \\
17 & 62,472 & 34.1 & 86.5 & 13.5 & 95.0 \\
18 & 63,725 & 35.4 & 86.2 & 13.8 & 94.7 \\
19 & 64,978 & 36.6 & 85.7 & 14.3 & 94.2 \\
20 & 66,231 & 37.8 & 85.5 & 14.5 & 94.0 \\
\hline
\end{tabular}

a Calculated number-average molecular weight of 4-arm PEG-An (DMT-off, phosphate and benzoyl deprotected).

${ }^{b}$ Integral of PEG-An in the HPLC trace divided by the integral of PEG- $A_{n}+$ oligo-A impurities.

${ }^{c}$ Integral of oligo-A impurities in the HPLC trace divided by the integral of PEG- $A_{n}+$ oligo-A impurities.

${ }^{d}$ Integral of PEG-An in the HPLC trace divided by the integral of PEG- $A_{n}+$ oligo-A impurities, after the integral of PEG- $A_{n}$ is divided by the DNA mass percentage of PEG- $A_{n}$ in the respective step. This assumes the extinction coefficient of $A_{n}$ scales linearly with $n$. 
Table S8. Comparison of the A-to-PEG ratio obtained from ${ }^{1} \mathrm{H}-\mathrm{NMR}$ with the 4-arm PEG-An purity obtained after HPLC purification to determine the actual number of $A$ nucleotides coupled to the 4-arm PEG.

\begin{tabular}{ccccc}
\hline Step & $\begin{array}{c}\text { NMR apparent } \\
\text { number of A per } \\
\text { PEG-arm }{ }^{\mathrm{a}}\end{array}$ & $\begin{array}{c}\text { HPLC molar } \\
\text { ratio PEG- } A_{n} \\
(\%)^{\mathrm{b}}\end{array}$ & $\begin{array}{c}\text { number of A per } \\
\text { PEG-arm } \\
(\%)^{\mathrm{c}}\end{array}$ & $\begin{array}{c}\text { total coupling } \\
(\%)^{\mathrm{d}}\end{array}$ \\
\hline 1 & 0.98 & 97.7 & 0.95 & 95.3 \\
2 & 1.98 & 98.6 & 1.95 & 97.6 \\
3 & 2.98 & 99.0 & 2.95 & 98.3 \\
4 & 3.97 & 98.5 & 3.91 & 97.6 \\
5 & 4.91 & 98.3 & 4.83 & 96.5 \\
6 & 5.94 & 98.1 & 5.83 & 97.1 \\
7 & 7.05 & 97.5 & 6.87 & 98.1 \\
8 & 7.99 & 96.8 & 7.73 & 96.7 \\
9 & 9.05 & 95.6 & 8.65 & 96.1 \\
10 & 10.09 & 94.2 & 9.50 & 95.0 \\
11 & 11.10 & 91.9 & 10.20 & 92.7 \\
12 & 12.19 & 90.6 & 11.04 & 92.0 \\
13 & 13.23 & 89.8 & 11.88 & 91.4 \\
14 & 14.39 & 88.5 & 12.74 & 91.0 \\
15 & 15.40 & 87.4 & 13.46 & 89.7 \\
16 & 16.49 & 87.6 & 14.44 & 90.3 \\
17 & 17.48 & 86.5 & 15.12 & 88.9 \\
18 & 18.53 & 86.2 & 15.97 & 88.7 \\
19 & 19.61 & 85.7 & 16.81 & 88.5 \\
20 & 20.55 & 85.5 & 17.57 & 87.8 \\
\hline
\end{tabular}

a The integral of A-Bz-2H compared to the $935 \mathrm{H}$ of the PEG-core divided by 2, see Table S6.

${ }^{b}$ Integral of PEG-An in the HPLC trace divided by the integral of PEG-An + oligo-A impurities, see Table S7.

c Obtained by multiplying the apparent number of A-nucleotides per arm (NMR) by the PEG-An purity.

d Number of $A$ nucleotides per arm divided by the number of reaction steps. 
NMR characterization of HPLC-purified 4-arm PEG-A ${ }_{10,15,20}$
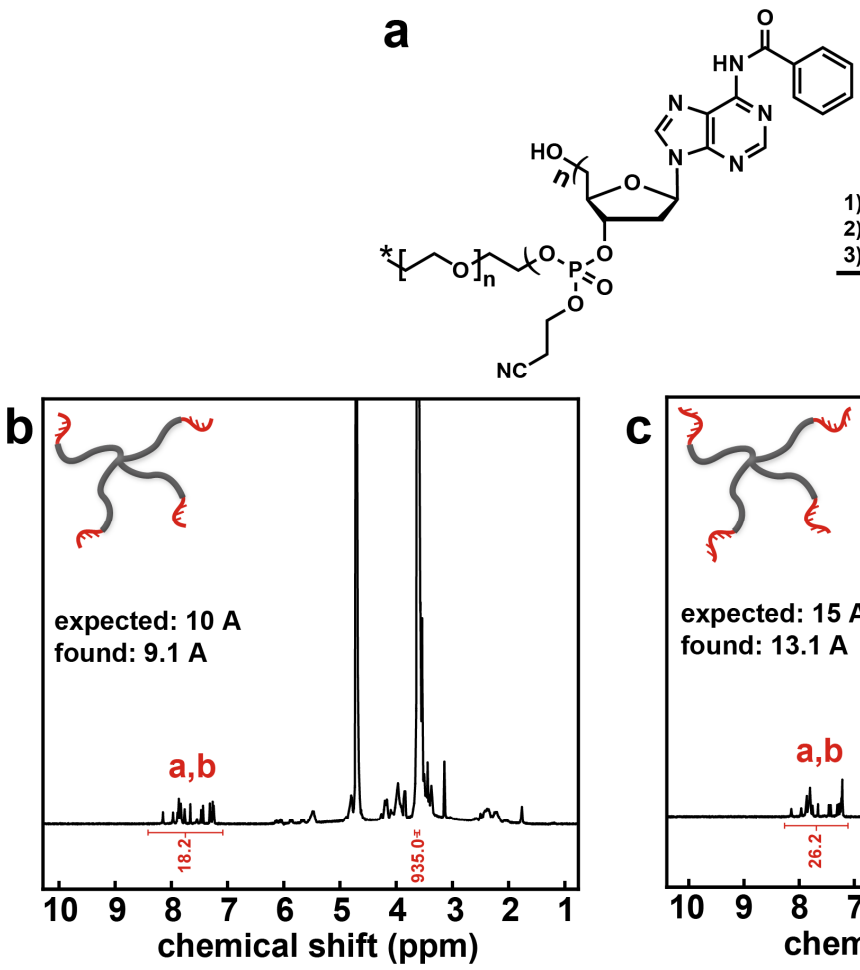

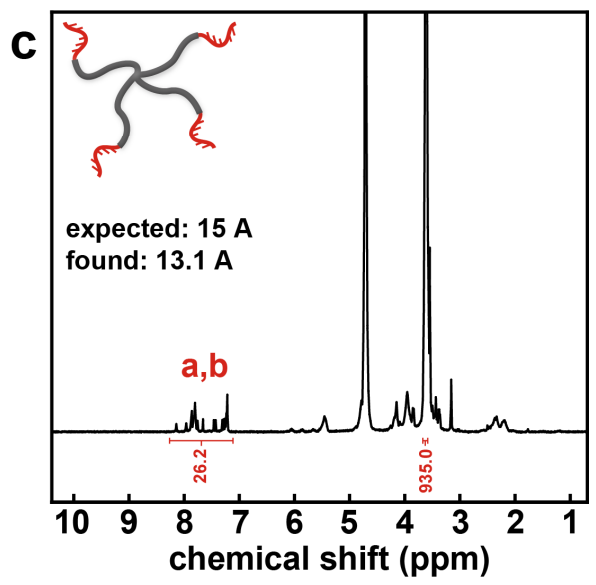

deprotection 2) HPLC-purification 3) dialysis
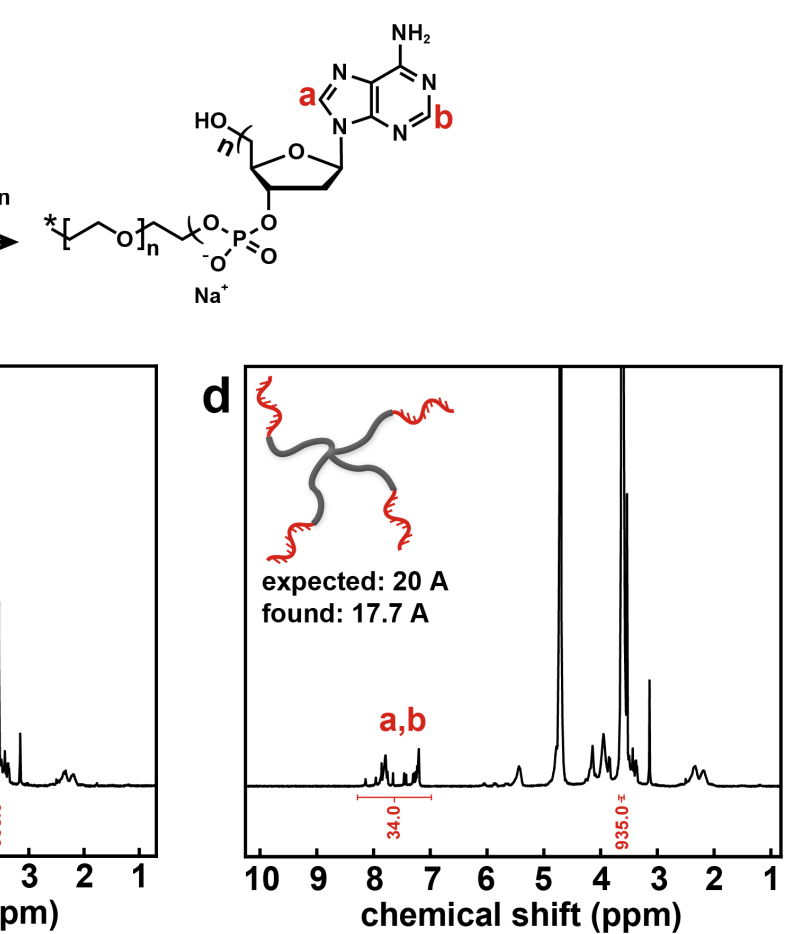

Figure S25. ${ }^{1} \mathrm{H}-\mathrm{NMR}$ characterization of 4 -arm PEG-A $\mathrm{A}_{10},-\mathrm{A}_{15}$ and $-\mathrm{A}_{20}$ after preparative HPLC purification. (a) Reaction scheme for the deprotection of the phosphate and adenine by removal of the CE- and benzoyl groups (120 minutes in ammonia/methylamine (AMA) solution) followed by preparative HPLC purification and dialysis in phosphate buffer to exchange the triethylammonium counterions from the HPLC buffer to sodium ions. (b-d) ${ }^{1} \mathrm{H}-\mathrm{NMR}\left(\mathrm{D}_{2} \mathrm{O}, 300 \mathrm{MHz}\right)$ of 4-arm PEG-A $\mathrm{A}_{10},-\mathrm{A}_{15}$ and $-\mathrm{A}_{20}$ after purification, showing the actual numbers of $A$ nucleotides attached per PEG-arm as determined by comparing the adenine-2H integral to the $935 \mathrm{H}$ integral of a single PEG arm. * represents the core of the 4-arm PEG. 


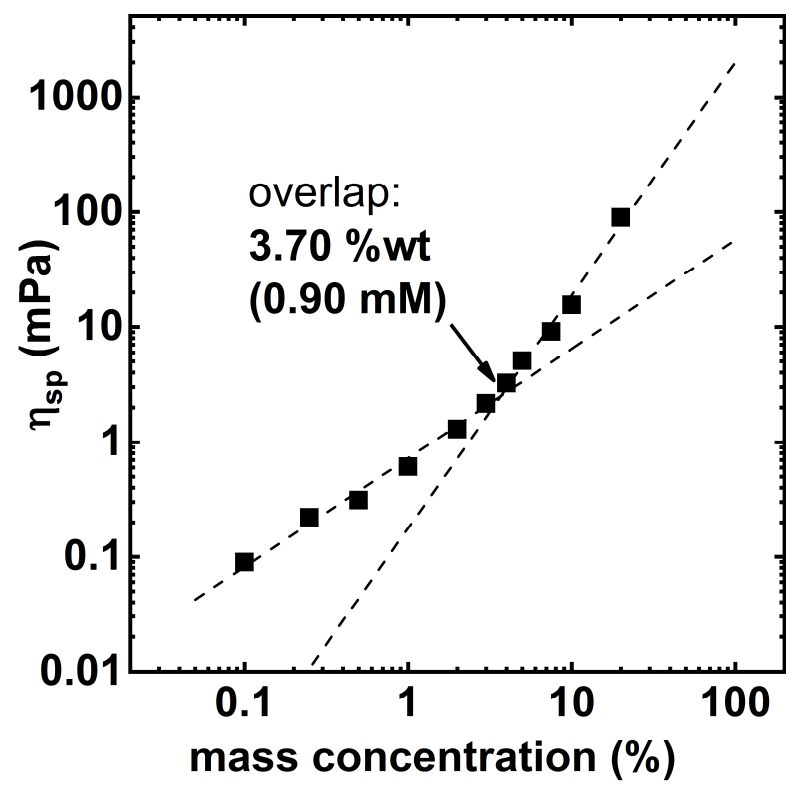

Figure S26. Overlap concentration for 4-arm PEG-OH $\left(M_{\mathrm{n}, \mathrm{MALDI}}=41 \mathrm{~kg} / \mathrm{mol}\right)$ determined as the crossover of two different slopes in the double logarithmic plot of the specific viscosity against the mass concentration in water. 